ARTICLE

\title{
Acetate functions as an epigenetic metabolite to promote lipid synthesis under hypoxia
}

Xue Gao ${ }^{1,2, \star}$, Shu-Hai Lin ${ }^{3,4, \star}$, Feng Ren ${ }^{1}$, Jin-Tao Li ${ }^{1,2}$, Jia-Jia Chen ${ }^{1}$, Chuan-Bo Yao ${ }^{1}$, Hong-Bin Yang ${ }^{1}$,

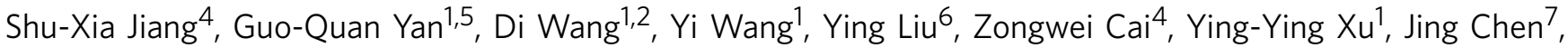
Wenqiang $\mathrm{Yu}^{1}$, Peng-Yuan Yang ${ }^{1} \&$ Qun-Ying Lei ${ }^{1}$

Besides the conventional carbon sources, acetyl-CoA has recently been shown to be generated from acetate in various types of cancers, where it promotes lipid synthesis and tumour growth. The underlying mechanism, however, remains largely unknown. We find that acetate induces a hyperacetylated state of histone $\mathrm{H} 3$ in hypoxic cells. Acetate predominately activates lipogenic genes ACACA and FASN expression by increasing H3K9, H3K27 and H3K56 acetylation levels at their promoter regions, thus enhancing de novo lipid synthesis, which combines with its function as the metabolic precursor for fatty acid synthesis. Acetyl-CoA synthetases (ACSS1, ACSS2) are involved in this acetate-mediated epigenetic regulation. More importantly, human hepatocellular carcinoma with high ACSS1/2 expression exhibit increased histone $\mathrm{H} 3$ acetylation and FASN expression. Taken together, this study demonstrates that acetate, in addition to its ability to induce fatty acid synthesis as an immediate metabolic precursor, also functions as an epigenetic metabolite to promote cancer cell survival under hypoxic stress.

\footnotetext{
${ }^{1}$ Key Laboratory of Metabolism and Molecular Medicine, Ministry of Education, and Department of Biochemistry and Molecular Biology, School of Basic Medical Sciences, and Institutes of Biomedical Sciences, Fudan University, Shanghai 200032, China. ${ }^{2}$ School of Life Science, Fudan University, Shanghai 200433, China. ${ }^{3}$ Department of Biochemistry and Molecular Cell Biology, Shanghai Key Laboratory for Tumor Microenvironment and Inflammation, Shanghai Jiao Tong University School of Medicine (SJTU-SM), Shanghai 200025, China. ${ }^{4}$ State Key Laboratory of Environmental and Biological Analysis, Department of Chemistry, Hong Kong Baptist University, Hong Kong 999077, China. ${ }^{5}$ Department of Chemistry, Fudan University, Shanghai 200032, China.

${ }^{6}$ Department of Pathology, School of Basic Medical Sciences, Fudan University, Shanghai 200032, China. ${ }^{7}$ Department of Hematology and Medical Oncology, Winship Cancer Institute of Emory, Emory University School of Medicine, Atlanta, Georgia 30322, USA. * These authors contributed equally to this work. Correspondence and requests for materials should be addressed to P.Y.Y. (email: pyyang@fudan.edu.cn) or to Q.Y.L. (email: qlei@fudan.edu.cn).
} 
A cetyl-CoA, as a central metabolic intermediate, is widely used in macromolecule biosynthesis and energy production to support cell growth and proliferation. As a donor of acetyl group, acetyl-CoA is also dynamically associated with acetylation modification to modulate protein functions. Therefore, maintenance of cellular acetyl-CoA pool is essential for the regulation of various cellular processes.

In human, acetyl-CoA is mainly produced from oxidation of glucose and other conventional carbon sources, such as glutamine and fatty acids ${ }^{1,2}$. However, in human brain cancers, glucose contributes $<50 \%$ carbons to cellular acetyl-CoA pool, suggesting the existence of a substitutive supply for acetyl$\mathrm{CoA}^{3}$. Subsequent studies reveal that cancer cells avidly capture acetate as their alternative carbon source to support cell survival and proliferation under stressed conditions, in particular hypoxia $^{3-9}$. Moreover, various human cancers show enhanced acetate uptake in $\left[{ }^{11} \mathrm{C}\right]$-acetate PET studies ${ }^{10-15}$. These findings suggest that cancer cells utilize acetate as an alternative carbon source to glucose to maintain cellular acetyl-CoA pool under stressed conditions.

Acetate has long been identified as a major carbon source in bacteria and yeasts. Yeast acetyl-CoA synthetases (Acs1p and Acs2p) fuel cell growth by converting acetate to acetyl-CoA ${ }^{16}$. Very recently, acetate is also found to be an alternative carbon source besides glucose, glutamine and fatty acids in human cancer, attracting intensive investigations ${ }^{7,8}$. Generally, mammalian acetyl-CoA synthesis from acetate is carried out by ACSS2 to support lipid synthesis in the cytosol, and by ACSS1 to fuel ATP production in mitochondria ${ }^{17-19}$. Acetate is mainly acquired from diet, but can also be generated in ethanol metabolism or deacetylation processes. The function of acetate has long been overlooked due to its relative low physiological concentration $(0.2-0.3 \mathrm{mM})$ in blood $^{20}$. Recent studies reveal that cancer cells show increased acetate uptake under hypoxia even in the presence of low acetate concentration to support tumour growth ${ }^{7,9,14}$. However, how cancer cells utilize acetate under hypoxia in such an efficient manner remains unclear.

Histone acetylation is intimately coordinated with cellular acetylCoA pool in response to metabolic state. As the downstream metabolite of carbon sources, acetyl-CoA represents a pivotal metabolic signal of nutrient availability ${ }^{4,21,22}$. In yeast, histone is specifically acetylated at genes involved in lipogenesis, aminoacid biosynthesis and cell cycle progression upon entry into growth, in tune with intracellular acetyl-CoA level ${ }^{23}$. ATP citrate lyase (ACLY), the enzyme converting glucose-derived citrate into acetyl-CoA, regulates histone acetylation by sensing glucose availability 1,22 . Yeast acetyl-CoA Carboxylase (Acc1p) consumes acetyl-CoA to synthesize lipids and regulates global histone acetylation through competing for the same nucleocytosolic acetyl-CoA pool ${ }^{24}$. Thus, the acetyl-CoA flux dynamically regulates gene expression profile by modulating histone acetylation state.

These observations led us to hypothesize that acetate induces a metabolic adaptation through modulating histone acetylation in hypoxic cancer cells. Consistent with this idea, we found that acetate predominately activates the expression of lipogenic genes through upregulating histone acetylation at their promoter regions, which in turn promotes lipid synthesis under hypoxia. Beyond a carbon source for macromolecular biosynthesis, our findings highlight an epigenetic role for acetate in metabolic adaptation of cancer cells to hypoxic stress.

\section{Results}

Acetate restores histone acetylation under hypoxia. Cancer cells demand distinctive extracellular nutrients and reprogram the metabolic pathways to survive and proliferate when facing harsh situation, such as hypoxia ${ }^{25}$. Hypoxia stress is a critical player in tumorigenesis and tumour development ${ }^{26}$. By performing exometabolome analysis based on ${ }^{1} \mathrm{H}-\mathrm{NMR}$ spectra, we found that cancer cells absorbed around 20\% acetate from the culture medium under normoxia while more than $80 \%$ acetate was consumed under hypoxia (Fig. 1a; Supplementary Fig. 1a,b), suggesting that cancer cells take up more acetate under hypoxia than nomoxia $7,9,14$. Moreover, we carried out the quantification of acetate from five pairs of hepatocellular carcinoma (HCC) and adjacent samples by NMR. As shown in Supplementary Fig. 1c, acetate concentration range was from 0.56 to $2.67 \mu \mathrm{molg}^{-1}$ in wet tissue (left) and acetate concentration in tissue would be roughly estimated around from 0.56 to $2.67 \mathrm{mM}$ (right). In most cases acetate concentrations ranged around $0.5 \mathrm{mM}$ and in two HCC samples acetate levels reached $2.5 \mathrm{mM}$ (Supplementary Fig. 1c). To explore acetate effect on cancer cell, we treated HepG2 cells with acetate under hypoxia and found that acetate counteracted the declined histone acetylation under hypoxia compared with normoxia (Fig. 1b). Of particular interest, acetate induced significant increase of $\mathrm{H} 3 \mathrm{~K} 9, \mathrm{H} 3 \mathrm{~K} 27$ and $\mathrm{H} 3 \mathrm{~K} 56$ acetylation levels, but not H3K14, H3K18, H3K23 and H3K36 acetylation levels (Fig. 1b). This indicates that acetate rescues hypoxia-reduced histone acetylation with certain specificity. More importantly, by using metabolic-labelling technique based on liquid chromatography tandem mass spectrometry in multiple reaction monitoring mode (LC-MRM MS), we identified ${ }^{13} \mathrm{C}_{2^{-}}$ labelled acetylated H3K9 (Supplementary Fig. 1d), H3K27 (Supplementary Fig. 1e) and H3K56 (Supplementary Fig. 1f) peptides in HepG2 cells treated with $\left[\mathrm{U}_{-}{ }^{13} \mathrm{C}_{2}\right]$-acetate, demonstrating that acetate-derived acetyl-CoA was indeed incorporated into histones. In addition, compared with normoxia, histone acetylation is more susceptible to acetate supplementation under hypoxia, even under low concentration, in HepG2, A549 and DU 145 cells (Fig. 1c; Supplementary Fig. 1g,h).

To characterize the manner of epigenetic regulation by acetate, we treated cancer cells with acetate for different time points under hypoxia. We found that exogenous acetate supplement rapidly increased $\mathrm{H} 3 \mathrm{~K} 9, \mathrm{H} 3 \mathrm{~K} 27$ and $\mathrm{H} 3 \mathrm{~K} 56$ acetylation levels in a timedependent manner under hypoxia in different cancer cell lines (Fig. 1d; Supplementary Fig. 1i,j). Acetate supplement dramatically increased $\mathrm{H} 3 \mathrm{~K} 9, \mathrm{H} 3 \mathrm{~K} 27$ and $\mathrm{H} 3 \mathrm{~K} 56$ acetylation levels within $2 \mathrm{~h}$ (Fig. 1d; Supplementary Fig. 1i,j). Furthermore, acetate was found to increase histone $\mathrm{H} 3 \mathrm{~K} 9, \mathrm{H} 3 \mathrm{~K} 27$ and $\mathrm{H} 3 \mathrm{~K} 56$ acetylation levels in a dose-dependent manner under hypoxia in HepG2 (Fig. 1e), A549 (Supplementary Fig. 1k), DU145 (Supplementary Fig. 1l), SkBr3 (Supplementary Fig. 1m) and HT29 cells (Supplementary Fig. 1n). Low concentration of acetate $(1.25 \mathrm{mM})$ slightly increased histone acetylation levels, while higher concentrations of acetate (2.5-5 mM) significantly increased histone acetylation levels under hypoxia (Fig. 1e; Supplementary Fig. 1k, l, m, n). Collectively, these data imply that acetate is implicated in the regulation of histone acetylation under hypoxia.

Acetate epigenetically activates de novo lipogenesis. It is well known that histone acetylation is associated with transcription activity $^{27-29}$. To investigate the biological effect on cancer metabolism induced by the acetate-mediated histone acetylation, we detected the mRNA expression changes of metabolic genes. We designed quantitative PCR (qPCR) primers targeting 139 metabolic genes, covering a wide range of metabolic pathways (that is, glycolysis, TCA cycle, pentose phosphate pathway, glycogen metabolism, ketone body metabolism, aminoacid metabolism, fatty acid synthesis and $\beta$ - 


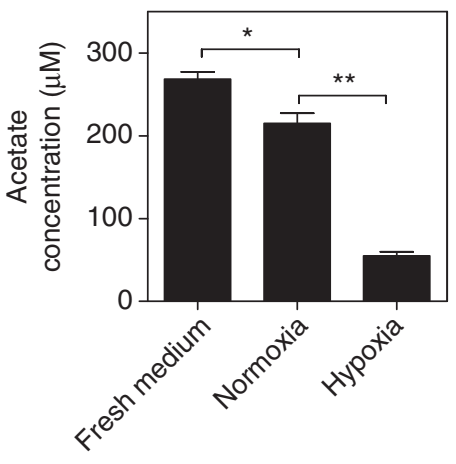

b

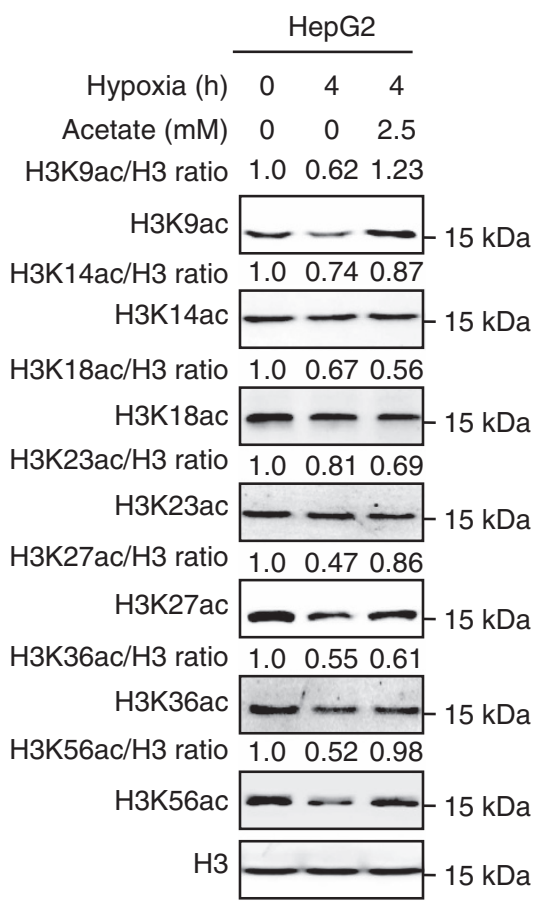

C

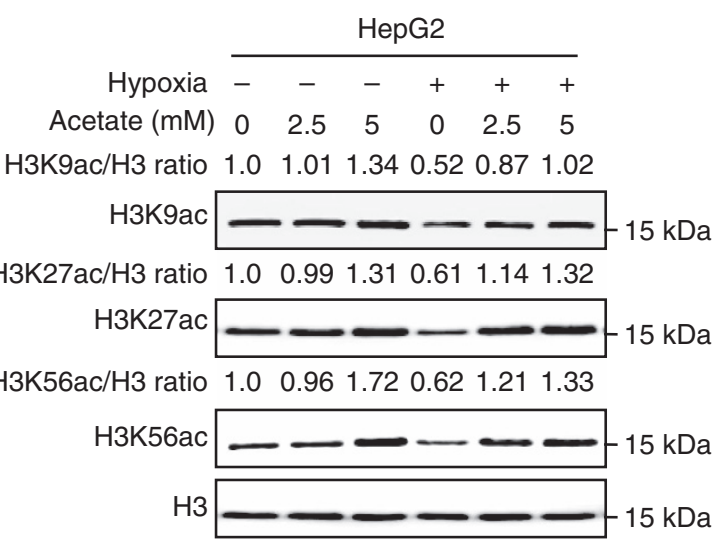

d

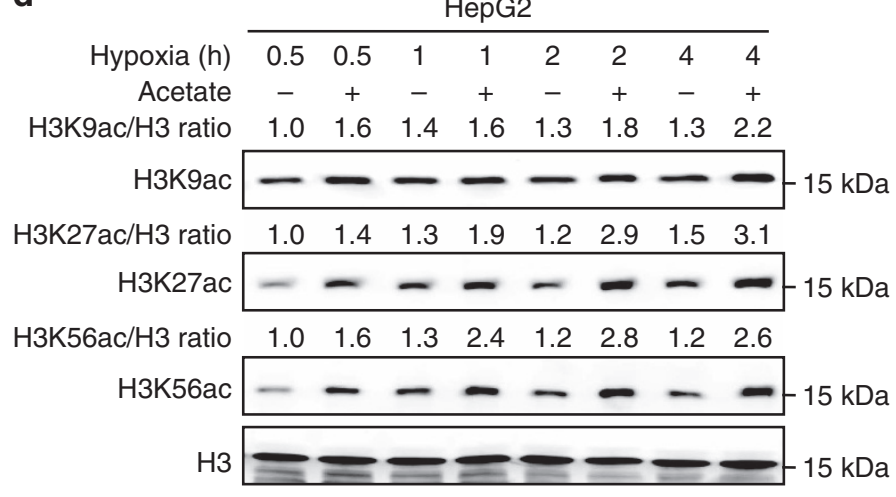

e

\begin{tabular}{llllll} 
& \multicolumn{4}{c}{ HepG2 (hypoxia) } \\
\cline { 2 - 5 } Acetate (mM) & 0 & 0.5 & 1.25 & 2.5 & 5
\end{tabular}

$\mathrm{H} 3 \mathrm{~K} 9 \mathrm{ac} / \mathrm{H} 3$ ratio $1.0 \quad 1.18 \quad 1.141 .671 .98$

H3K9ac $\longrightarrow-15 \mathrm{kDa}$

H3K27ac/H3 ratio $1.0 \quad 1.08 \quad 1.211 .451 .92$

H3K27ac - - - $15 \mathrm{kDa}$

H3K56ac/H3 ratio $1.0 \quad 0.86 \quad 0.93 \quad 1.32 \quad 1.93$

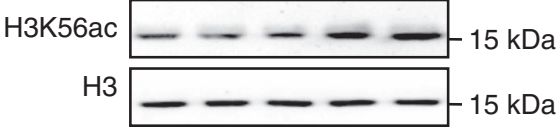

Figure 1 | Acetate rescues hypoxia-induced reduction of histone acetylation. (a) ${ }^{1} \mathrm{H}-\mathrm{NMR}$ spectra of acetate concentration in fresh medium, medium of cancer cells cultured under normoxia or hypoxia. The results represented mean \pm s.d. of triplicate experiments. ( ${ }^{\star \star} P<0.01$; ${ }^{\star} P<0.05$; by two-tailed unpaired Student's t-test). (b) Acetate rescues hypoxia-reduced H3K9, H3K27 and H3K56 acetylation levels. HepG2 cells were treated with or without 2.5 mM acetate under hypoxia $\left(1 \% \mathrm{O}_{2}\right)$ for $4 \mathrm{~h}$. The histone acetylation levels were determined by western blot. Total $\mathrm{H} 3$ served as a loading control. (c) Cancer cells are more sensitive to acetate under hypoxia. $\mathrm{HepG} 2$ cells were treated with indicated concentrations of acetate under normoxia or hypoxia $\left(1 \% \mathrm{O}_{2}\right.$ ) for $4 \mathrm{~h}$. The histone acetylation levels were determined by western blot. Total H3 served as a loading control. (d) Acetate increases H3K9, H3K27 and H3K56 acetylation levels in a time-dependent manner under hypoxia. HepG2 cells were treated with or without $5 \mathrm{mM}$ acetate for $0.5,1,2$ and $4 \mathrm{~h}$ under hypoxia (1\% $\mathrm{O}_{2}$ ), respectively. The global histone acetylation levels were determined by western blot. Total histone $\mathrm{H} 3$ served as a loading control. (e) Acetate increases H3K9, H3K27 and H3K56 acetylation levels in a dose-dependent manner under hypoxia. HepG2 cells were treated with the indicated concentrations of acetate for $4 \mathrm{~h}$ under hypoxia $\left(1 \% \mathrm{O}_{2}\right)$. The histone acetylation levels were determined by western blot. Total $\mathrm{H} 3$ served as a loading control.

oxidation, cholesterol synthesis, sphingolipid metabolism, and metabolism-related transporters) (Supplementary Table 1) and conducted qPCR to screen potential metabolic pathway(s) affected by acetate-induced epigenetic regulation. Scatter plot of mRNA expression data of 139 genes in HepG2 cells was shown in Fig. 2a, by comparing cell culture under hypoxia with normoxia. The expression of VEGF and $L D H A$, which were included as positive controls for verifying hypoxic effect, increased by more than two-fold under hypoxia (Fig. 2a). We then tested the effect of acetate on gene expression under either normoxia (Fig. 2b) or hypoxia (Fig. 2c), respectively. Compared with the normoxia group, the upregulated genes by two-fold in hypoxia group and hypoxia with acetate group showed a lot overlap (Supplementary Fig. 2a). Besides, FASN and ACSS2 were the unique genes in hypoxia with acetate group, indicating that acetate played an important role in lipid synthesis under hypoxia (Supplementary Fig. 2a). In line with this, FASN and ACACA mRNA levels were activated by more than two-fold with acetate under hypoxia, 
a

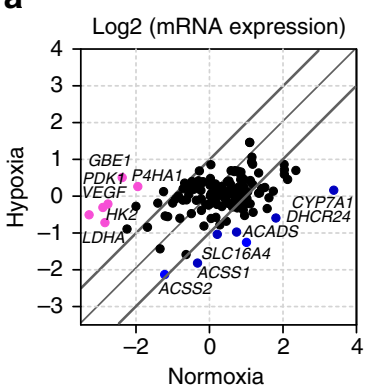

b

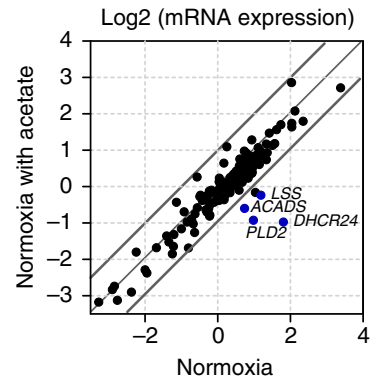

C

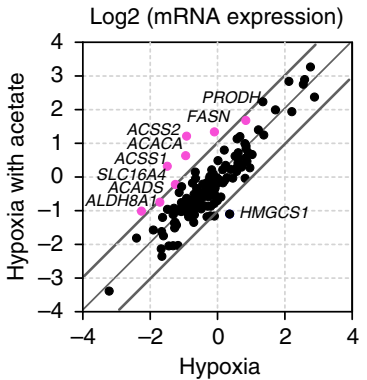

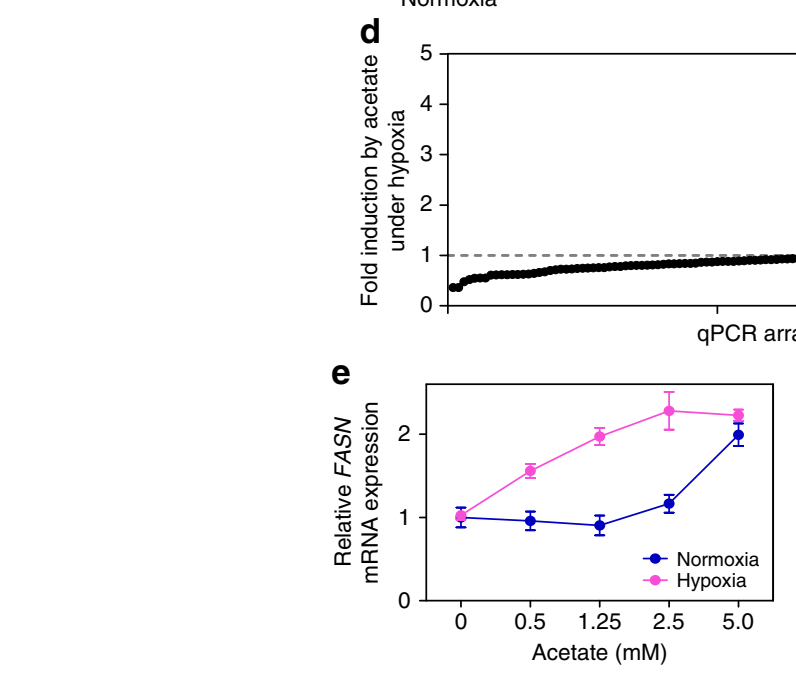
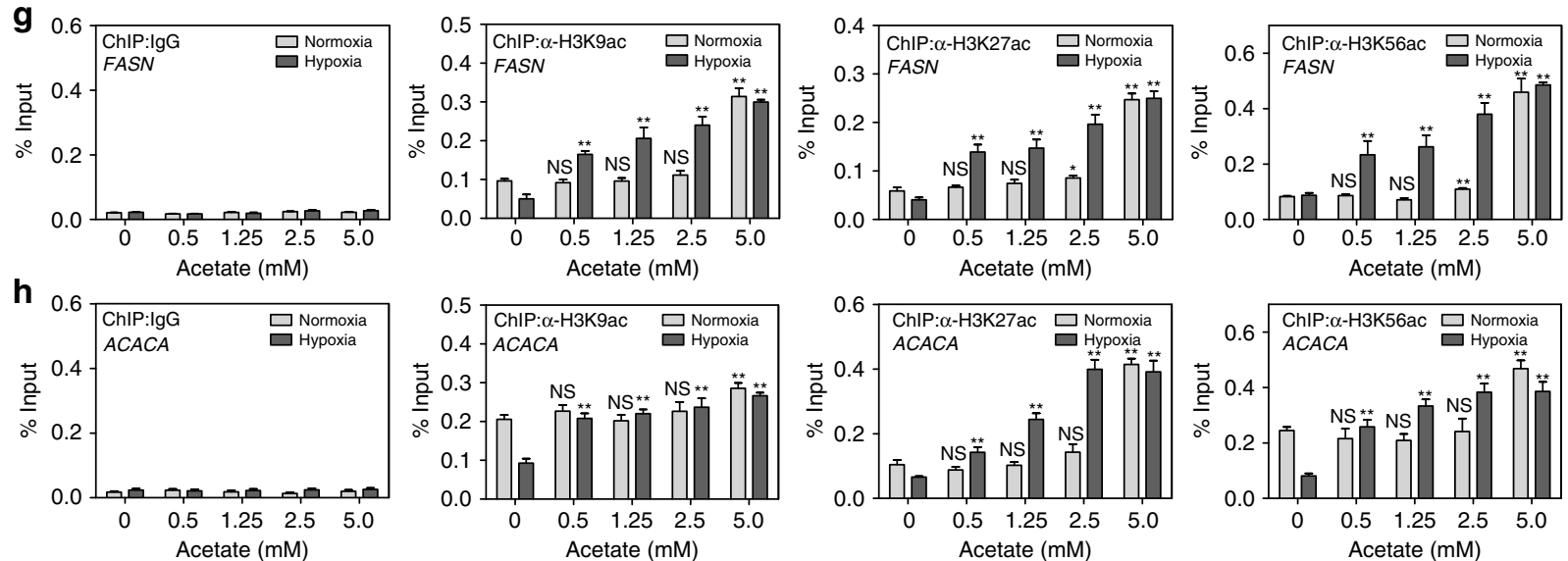

Figure 2 | Acetate predominately activates lipid synthesis pathway through epigenetic regulation under hypoxia. (a) Scatter plot of mRNA expression data of 139 metabolic genes in HepG2 cells, comparing cells treated with hypoxia ( $y$ axis) to normoxia ( $x$ axis). The mRNA expression value of triplicate experiments was shown on a log 2 scale. Grey lines indicated two-fold differences in the mRNA expression levels between two groups. Upregulated genes ( $>2$-fold change, $P<0.05$ ) were shown in magenta. Downregulated expressed genes ( $>2$-fold change, $P<0.05$ ) were shown in blue. Two-tailed unpaired Student's $t$-test was used. (b) Scatter plot of mRNA expression data of 139 metabolic genes in HepG 2 cells under normoxia, comparing cells treated with $2.5 \mathrm{mM}$ acetate ( $y$ axis) to acetate-free ( $x$ axis). The mRNA expression value of triplicate experiments was shown on a log2 scale. Grey lines indicated twofold differences in the mRNA expression levels between two groups. Downregulated genes ( $>2$-fold change, $P<0.05$ ) were shown in blue. Two-tailed unpaired Student's $t$-test was used. (c) Scatter plot of mRNA expression data of 139 metabolic genes in HepG2 cells under hypoxia, comparing cells treated with $2.5 \mathrm{mM}$ acetate $(y$ axis) with acetate-free ( $x$ axis). The mRNA expression value of triplicate experiments was shown on a log 2 scale. Grey lines indicated two-fold differences in the mRNA expression levels between two groups. Upregulated genes ( $>2$-fold change, $P<0.05$ ) were shown in magenta. Two-tailed unpaired Student's t-test was used. (d) Fold-change analysis of the mRNA expression of 139 genes in HepG2 cells treated with or without $2.5 \mathrm{mM}$ acetate under hypoxia. (e,f) FASN (e) and ACACA (f) mRNA levels in HepG2 cells treated with indicated concentrations of acetate for $12 \mathrm{~h}$ under normoxia or hypoxia were quantified by qPCR. The results were presented as mean \pm s.d. of triplicate experiments. (g,h) ChIP-qPCR assays showing H3K9, H3K27 and H3K56 acetylation enrichment at FASN (g) and ACACA (h) promoter regions in HepG2 cells treated with indicated concentrations of acetate under normoxia or hypoxia for $4 \mathrm{~h}$. Rabbit IgG was included as a negative control. Each histogram was presented as mean $\pm \mathrm{s}$.d. of triplicate experiments $\left({ }^{\star} P<0.05 ;{ }^{\star \star} P<0.01 ; N S\right.$, not significant; by two-tailed unpaired Student's $t$-test). 
while no activation effect was observed under normoxia (Fig. 2b,c). Both FASN and ACACA are in the top-10 list of upregulated metabolic genes induced by acetate under hypoxia, indicating that acetate predominantly affects de novo lipid synthesis compared with other metabolic pathways (Fig. 2c,d). Interestingly, ACSS1 and ACSS2 mRNA levels were decreased under hypoxia compared with normoxia (Fig. 2a), which was discrepant with the other studies reported that ACSS2 was upregulated under hypoxia ${ }^{9,14}$. This may be due to the experimental conditions, such as different cell lines used and treating time points. Moreover, mRNA level of ACSS1 and $A C S S 2$, rather than $A C L Y$, were also upregulated on acetate treatment under hypoxia (Fig. 2c,d). The rescue of hypoxiareduced ACSS1 and ACSS2 expression by acetate may indicate that ACSS1 and ACSS2 play an important role in cancer cells adapting to hypoxia.

We further investigated whether acetate-induced histone acetylation was associated with acetate-promoted gene transcription on lipogenesis. We treated cancer cells with gradient concentrations of acetate and found that FASN and ACACA mRNA expression were upregulated in a dose-dependent manner under hypoxia (Fig. 2e,f). Intriguingly, change in FASN and $A C A C A$ mRNA expression are more prone to low concentration of acetate under hypoxia, compared with normoxic control (Fig. 2e,f). To characterize whether the upregulation of lipogenic genes is caused by histone acetylation induced by acetate supplementation, we treated cancer cells with trichostatin A (TSA), a histone deacetylase inhibitor, as a positive control. We found that TSA and acetate increased both the global acetylation level of histone $\mathrm{H} 3$ and the mRNA expression of FASN and $A C A C A$, although TSA and acetate had no synergic effect (Supplementary Fig. 2b). In addition, acetate treatment led to time-dependent enhancement of $\mathrm{H} 3 \mathrm{~K} 9, \mathrm{H} 3 \mathrm{~K} 27$ and $\mathrm{H} 3 \mathrm{~K} 56$ acetylation levels (Supplementary Fig. 2c). In line with the increased histone $\mathrm{H} 3$ acetylation, FASN and ACACA mRNA expressions were also elevated in a time-dependent manner (Supplementary Fig. 2c). Furthermore, we carried out ChIP-qPCR assays to define the underlying mechanism of acetate-induced FASN and ACACA expressions. We found that acetate markedly enhanced $\mathrm{H} 3 \mathrm{~K} 56$ acetylation level at the promoters of FASN and ACACA (Supplementary Fig. 2d,e), but not ACLY (Supplementary Fig. 2f). Furthermore, we found that histone acetylation ( $\mathrm{H} 3 \mathrm{~K} 9 \mathrm{ac}, \mathrm{H} 3 \mathrm{~K} 27 \mathrm{ac}$ and $\mathrm{H} 3 \mathrm{~K} 56 \mathrm{ac})$ at the promoters of FASN and ACACA responded to acetate treatment in a dose-dependent manner and histone acetylation was more prone to be induced by low concentration $(\leq 2.5 \mathrm{mM})$ of acetate under hypoxia, compared with normoxia (Fig. 2g,h). These results demonstrate that acetate is capable of epigenetically regulating lipogenic genes at pathophysiological concentration.

To rule out the possibility that promoter acetylation of FASN and $A C A C A$ reflects increased transcription, we alternatively performed ChIP-qPCR and tested promoter histone acetylation of LDHA and VEGF (Supplementary Fig. 2g, h, i, j). The acetylation levels (H3K9ac, H3K27ac and $\mathrm{H} 3 \mathrm{~K} 56 \mathrm{ac}$ ) at $L D H A$ and VEGF promoters were increased under hypoxia, which was further upregulated by acetate supplementation (Supplementary Fig. 2g, h, i, j). Consistently, mRNA expression of $L D H A$ and $V E G F$ was also activated by acetate (Supplementary Fig. 2k,l). These observations suggest that acetate contributes to histone acetylation at the promoters of hypoxia-induced genes. Collectively, these data support our notion that acetate promotes lipid synthesis pathway through epigenetic regulation.

Acetate-induced lipogenesis does not reflect lipid demands. Cancer cells show increased demands for lipids, through scavenging extracellular lipids or de novo lipid synthesis ${ }^{2,30,31}$. We tried to figure out whether acetate-induced expression of FASN or ACACA is affected by the extracellular lipids under hypoxia. Consistent with data in Fig. 2, FASN and ACACA mRNA expression was more prone to be induced by acetate under hypoxia, compared with normoxia (Fig. 3a,c) and acetateactivated promoter histone acetylation of FASN and ACACA more pronouncedly under hypoxia (Fig. 3b,d). Furthermore, lipid depletion in the serum did not alter acetate-induced expression of FASN and ACACA in HepG2 cells (Fig. 3e; Supplementary Fig. 3a). Similarly, FASN and ACACA promoter histone acetylation was not affected by lipid depletion as well (Fig. 3f; Supplementary Fig. 3b). Moreover, palmitate supplementation did not change the expression of FASN, VEGF and ACACA (Fig. 3g; Supplementary Fig. 3c,d). Consistently, histone acetylation at the promoter regions of FASN and ACACA did not respond to palmitate supplementation (Fig. 3h; Supplementary Fig. 3e). These results demonstrate that acetate increases histone acetylation at promoters of lipogenic genes and activates their mRNA expression, without reflecting lipid demand of hypoxic cells.

ACSS1 and ACSS2 are both involved in acetate-induced lipogenesis. In mammals, two main enzymes involve in acetyl-CoA production from acetate: the cytosolic ACSS2 and its mitochondrial paralogue ACSS1. To dissect which enzyme is responsible for mediating the acetate-induced increase of histone acetylation, we knocked down ACSS1 or ACSS2 individually and assessed the corresponding histone acetylation levels. To our surprise, knockdown of either ACSS1 or ACSS2 was incapable of blocking acetate-induced increase in acetylation levels at H3K9, H3K27 and H3K56 sites (Supplementary Fig. 4a,b). Though discrepant with the other studies reporting that ACSS2, but not ACSS1, appeared to be the key enzyme involved in metabolizing acetate for lipid synthesis ${ }^{5,9}$, our findings were consistent with those by Yun et al. ${ }^{32}$ and Bjornson et al. ${ }^{33}$, which reported that ACSS1 was also vital for acetate-dependent lipid synthesis. These results indicate that ACSS1 and ACSS2 are functionally redundant. We then established ACSS1/2 double knockdown stable cell pools. Concurrent knockdown of ACSS1/2 by using two different sets of shRNAs dramatically inhibited acetate-induced increase in acetylation levels of $\mathrm{H} 3 \mathrm{~K} 9, \mathrm{H} 3 \mathrm{~K} 27$ and $\mathrm{H} 3 \mathrm{~K} 56$ under hypoxia (Fig. 4a; Supplementary Fig. 4c). Moreover, we conducted quantification based on LC-MRM MS to see whether ACSS1/2 double knockdown could reduce the abundance of histone acetylation peptides derived from acetate by using $\left[\mathrm{U}-{ }^{13} \mathrm{C}_{2}\right]-$ acetate tracer. Acetate-derived acetyl-CoA was found actively incorporated into $\mathrm{H} 3 \mathrm{~K} 9, \mathrm{H} 3 \mathrm{~K} 14$ and $\mathrm{H} 3 \mathrm{~K} 27$ acetylation sites, compared with $\mathrm{H} 3 \mathrm{~K} 18, \mathrm{H} 3 \mathrm{~K} 23$ and $\mathrm{H} 3 \mathrm{~K} 56$ acetylation sites (Fig. 4b; Supplementary Fig. 4d). These results demonstrate that acetate could function as an acetyl donor to induce histone acetylation. ACSS1/2 double knockdown induced a more significant decrease in ${ }^{13} \mathrm{C}_{2}$-labelled acetylated $\mathrm{H} 3 \mathrm{~K} 9, \mathrm{H} 3 \mathrm{~K} 27$ peptides derived from $\left[\mathrm{U}_{-}{ }^{13} \mathrm{C}_{2}\right]$-acetate (Fig. $4 \mathrm{~b}$; Supplementary Fig. 4d). As for the discrepancy of H3K56 acetylation between western blot data and MS quantification, it might be due to the fact that concentration of ${ }^{13} \mathrm{C}_{2}$-labelled acetylated $\mathrm{H} 3 \mathrm{~K} 56$ peptide was too low to meet the limit of quantification. In addition, we designed experiments to test whether ACSS1/2 double knockdown could block acetate-induced histone acetylation at FASN and ACACA promoter regions. ACSS1/2 knockdown efficiency was verified by qPCR (Supplementary Fig. 4e). We found that acetate-increased $\mathrm{H} 3 \mathrm{~K} 9, \mathrm{H} 3 \mathrm{~K} 27$ and $\mathrm{H} 3 \mathrm{~K} 56$ acetylation levels at FASN (Fig. 4c) and ACACA (Fig. 4d) promoter regions were blocked by $A C S S 1 / 2$ double knockdown 
a

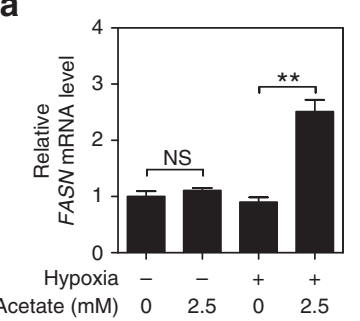

b
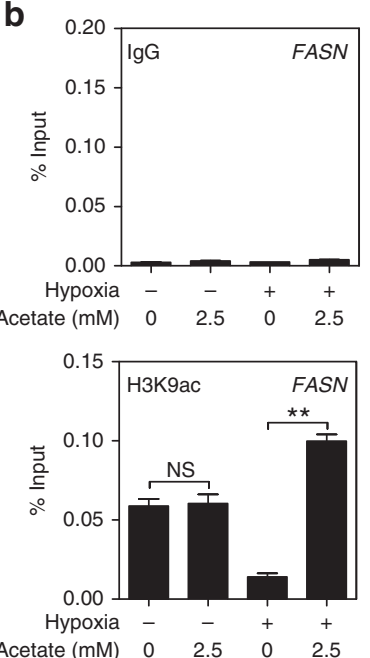

Acetate $(\mathrm{mM}) \quad 0 \quad 2.5 \quad 0 \quad 2.5$
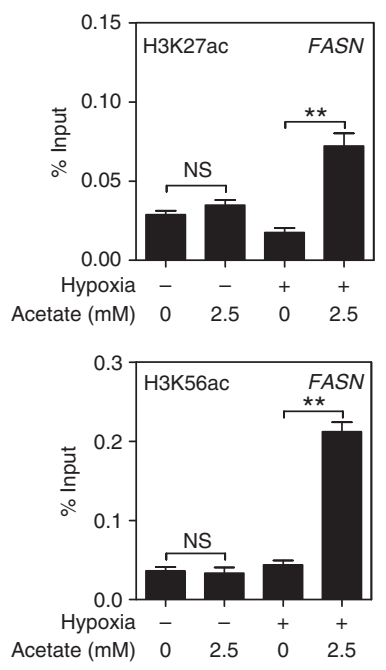

C

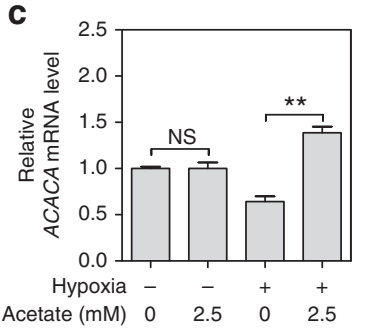

d
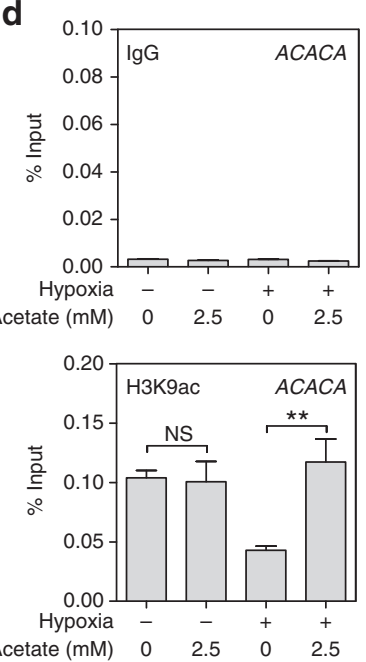

Acetate $(\mathrm{mM}) \quad 0 \quad 2.5 \quad 0 \quad 2.5$

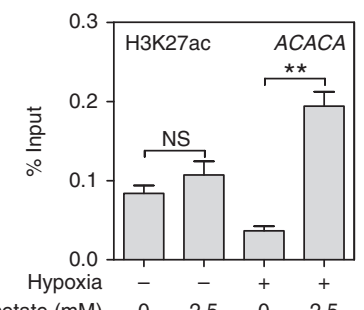

Acetate $(\mathrm{mM}) \quad 0 \quad 2.5 \quad 0 \quad 2.5$

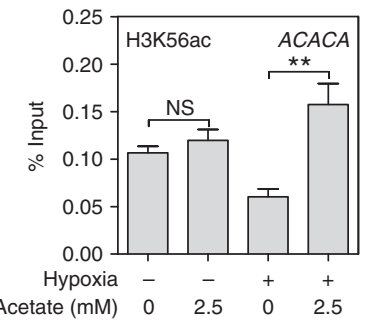

e

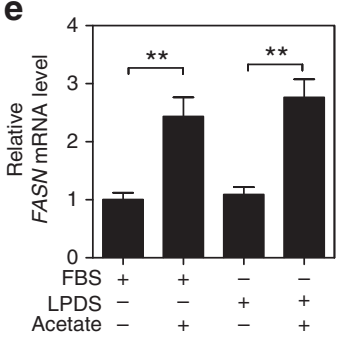

f
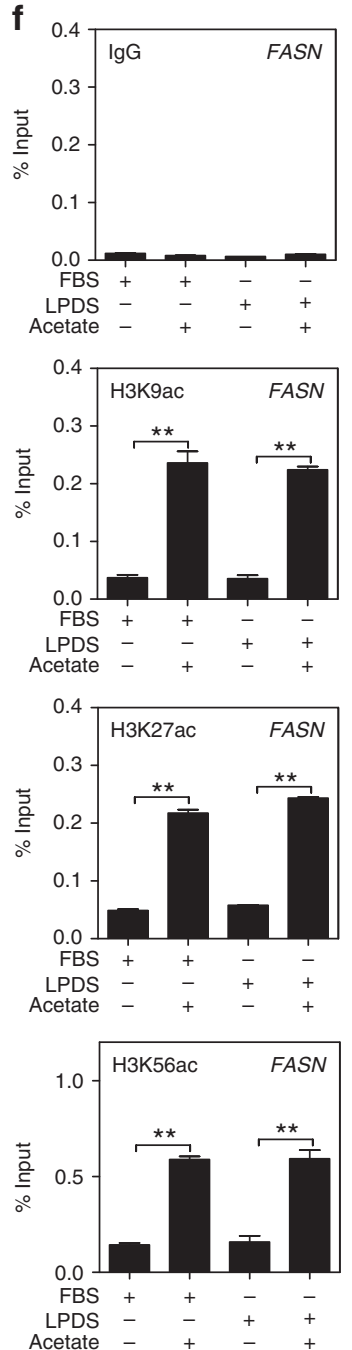

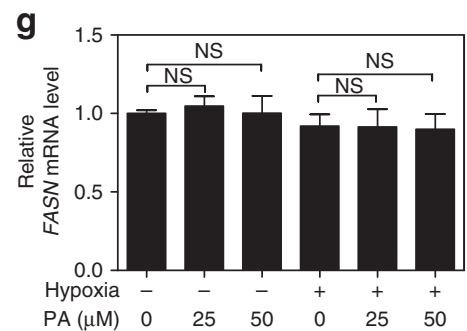

h
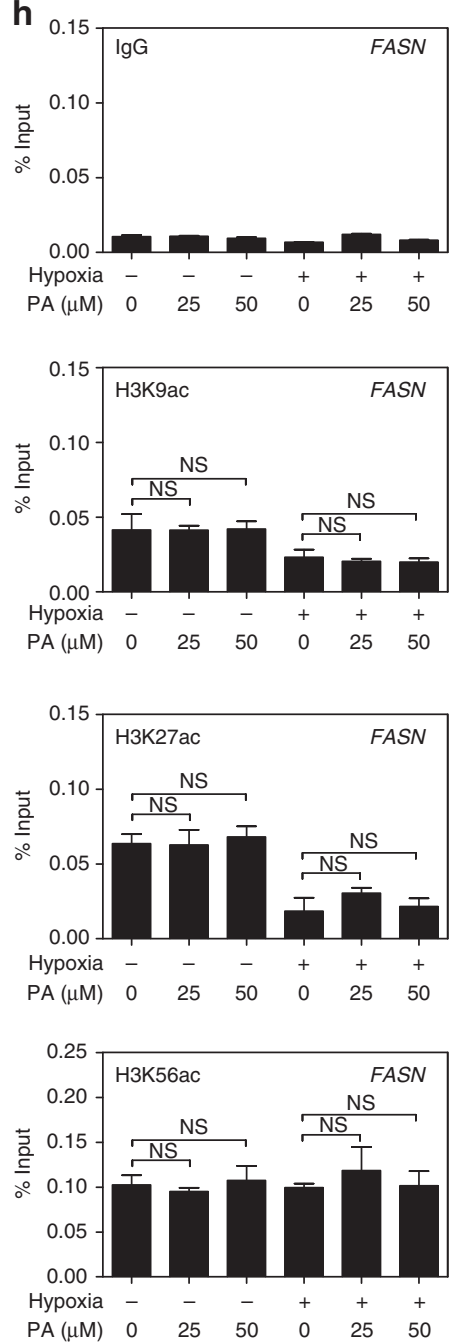

Figure 3 | Acetate epigenetically activates lipogenic genes without reflecting cellular lipid demands. (a) FASN expression in HepG2 cells treated with indicated concentrations of acetate for $12 \mathrm{~h}$ under normoxia or hypoxia were quantified by qPCR. The results were presented as mean $\pm \mathrm{s}$.d. of triplicate experiments ( ${ }^{\star *} P<0.01$; NS, not significant; by Student's $t$-test). (b) ChIP-qPCR assays showing histone acetylation enrichment at $F A S N$ promoter region in HepG2 cells treated with indicated concentrations of acetate under normoxia or hypoxia for $4 \mathrm{~h}$. Rabbit lgG was included as a negative control. Each histogram was presented as mean \pm s.d. of triplicate experiments ( ${ }^{\star *} P<0.01$; NS, not significant; by Student's t-test). (c) ACACA expression in HepG2 cells treated as in panel (a) were quantified by qPCR. The results were presented as mean \pm s.d. of triplicate experiments $\left({ }^{\star \star} P<0.01 ; N S\right.$, not significant; by Student's t-test). (d) ChIP-qPCR assays showing histone acetylation enrichment at ACACA promoter region in HepG2 cells treated as in $\mathbf{b}$. Each histogram was presented as mean \pm s.d. of triplicate experiments ( ${ }^{\star *} P<0.01$; NS, not significant; by Student's $t$-test). (e) Quantification of FASN expression in HepG2 cells treated with or without $2.5 \mathrm{mM}$ acetate in media plus $10 \%$ FBS or $10 \%$ LPDS for $12 \mathrm{~h}$ under hypoxia by qPCR. The results were presented as mean \pm s.d. of triplicate experiments ( ${ }^{\star \star} P<0.01$; by Student's $t$-test). (f) $C$ hIP-qPCR assays showing histone acetylation levels at FASN promoter region in HepG2 cells treated as in e for $4 \mathrm{~h}$ under hypoxia. The results were presented as mean \pm s.d. of triplicate experiments $\left({ }^{\star \star} P<0.01\right.$; by Student's $t$-test). (g) Quantification of FASN expression in HepG2 cells treated with $10 \%$ LPDS plus the indicated concentration of palmitate (PA) for $12 \mathrm{~h}$ under normoxia or hypoxia by qPCR. The results were presented as mean \pm s.d. of triplicate experiments (NS, not significant; by Student's t-test). (h) ChIP-qPCR assays showing histone acetylation levels enrichment at FASN promoter region in HepG2 cells treated as in $\mathbf{g}$ for $4 \mathrm{~h}$ under normoxia or hypoxia. The results were presented as mean \pm s.d. of triplicate experiments (NS, not significant; by Student's $t$-test). 
a

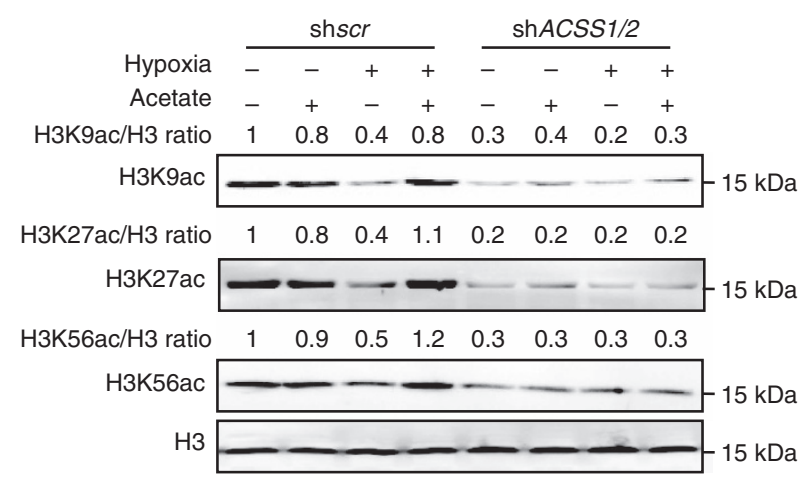

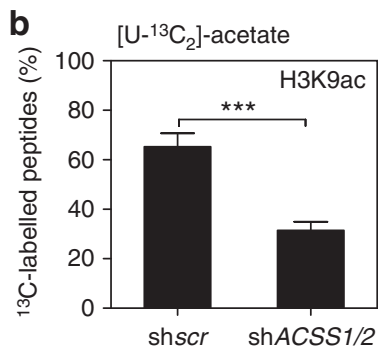
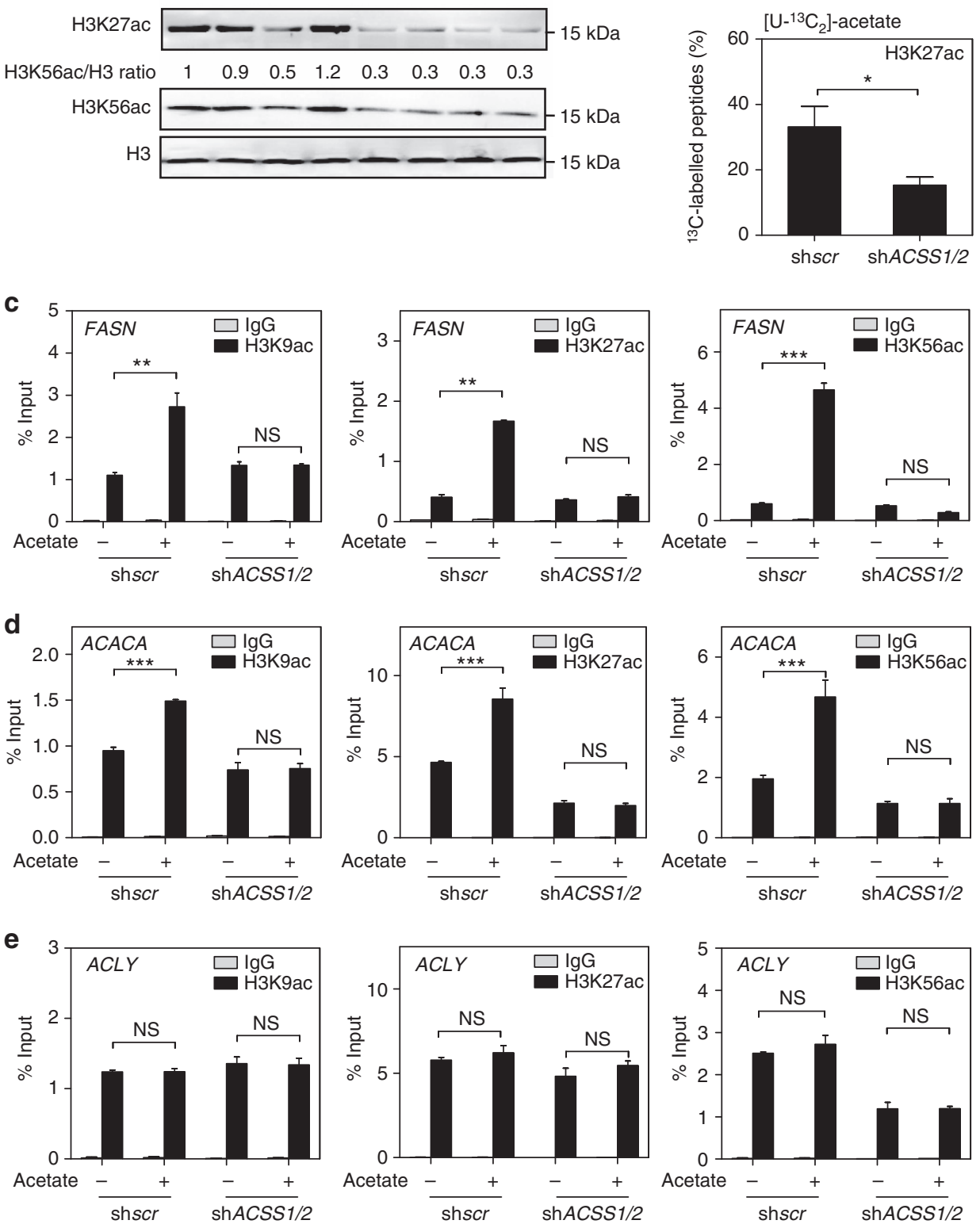

Figure 4 | Both ACSS1 and ACSS2 are involved in acetate-induced epigenetic regulation of de novo lipogenesis. (a) Stably concurrent knockdown of ACSS1 and ACSS2 totally blocks acetate-induced increase of histone acetylation levels. Histone acetylation levels in shscr or shACSS1/2 HepG2 cells treated with or without $2.5 \mathrm{mM}$ acetate under normoxia or hypoxia were analysed by western blot. $(\mathbf{b}){ }^{13} \mathrm{C}_{2}$-labelled $\mathrm{H} 3 \mathrm{~K} 9 \mathrm{ac}$ and $\mathrm{H} 3 \mathrm{~K} 27 \mathrm{ac}$ levels are decreased by ACSS1/2 knockdown. Quantification of ${ }^{13} \mathrm{C}_{2}$-labelled histone $\mathrm{H} 3$ acetylation levels in shscr or shACSS1/2 HepG2 stable cell line treated with [U- ${ }^{13} \mathrm{C}_{2}$ ]acetate for $4 \mathrm{~h}$ under hypoxia was analysed by LC-MRM MS. The percentage indicates the ratio of $\mathrm{H} 3 \mathrm{~K}\left[{ }^{13} \mathrm{C}_{2}\right.$-ac $] /$ total $\mathrm{H} 3 \mathrm{Kac}$ in each site. The results were presented as mean \pm s.d. of triplicate experiments $\left({ }^{\star} P<0.05 ;{ }^{\star \star \star} P<0.001\right.$; by Student's $t$-test). (c,d) ACSS1/2 knockdown totally blocks acetate-induced histone acetylation association with FASN and ACACA promoter. ChIP-qPCR assays were performed to determine H3K9, H3K27 and H3K56 acetylation enrichment at FASN (c) and ACACA (d) promoter region in shscr or shACSS1/2 HepG2 stable cell lines treated with or without acetate under hypoxia for 4h. IgG was included as a negative control. The results were presented as mean \pm s.d. of triplicate experiments $\left({ }^{\star \star} P<0.01 ;{ }^{* \star} P<0.001 ; N S\right.$, not significant; by Student's t-test). (e) ChIP-qPCR assays were performed to determine H3K9, H3K27 and H3K56 acetylation enrichment at ACLY promoter region in shsCr or shACSS1/2 HepG2 cells treated as in c. IgG was included as a negative control. The results were presented as mean \pm s.d. of triplicate experiments (NS, not significant; by Student's t-test). 
under hypoxia. ACSS1/2 knockdown had no effect on histone acetylation levels at ACLY promoter region (Fig. 4e). Consistently, ACSS1/2 knockdown blocked acetate-induced FASN expression (Supplementary Fig. 4f). Collectively, our data demonstrate that acetate contributes to histone acetylation in an ACSS1/2-dependent manner under hypoxia.

Previous study linked ACLY-dependent glucose metabolism to histone acetylation ${ }^{1,22}$. To dissect contributions of different carbon sources to histone acetylation, we performed qPCR and ChIP-qPCR assays in ACSS1/2-knockdown and/or ACLYknockdown cells. The knockdown efficiency was verified (Supplementary Fig. 4g). Depletion of either ACSS1/2 or ACLY reduced gene expression of $L D H A$ and $V E G F$, which were further decreased by knockdown of both ACSS1/2 and ACLY (Supplementary Fig. 4h,i). Consistently, either ACSS1/2 or $A C L Y$ knockdown reduced histone acetylation at the promoter regions of $L D H A$ and VEGF (Supplementary Fig. 4j, k, l, m). These results demonstrate that both ACSS1/2 and ACLY contribute to histone acetylation under hypoxia.

Acetate-induced lipogenesis promotes cell survival. To test whether acetate would affect lipid pool in cancer cells, we cultured HepG2 stable cells in media with isotope-labelled [ $\left.\mathrm{U}_{-}{ }^{13} \mathrm{C}_{2}\right]-$ acetate for $24 \mathrm{~h}$ under hypoxia and measured the isotopologues of fatty acids pool by using gas chromatography coupled with mass spectrometry (GC-MS). ACSS1/2 double knockdown indeed decreased the fractional contribution of acetate to palmitate and stearate synthesis (Fig. 5a). These results are consistent with the model that acetyl-CoA derived from acetate stimulates de novo lipid synthesis ${ }^{5,7,9}$. Furthermore, we knocked down FASN expression to the unstimulated state by using the low-efficiency siRNA as in Supplementary Fig. 5a, and treated cells with isotopelabelled $\left[\mathrm{U}_{-}{ }^{13} \mathrm{C}_{2}\right]$-acetate under hypoxia. The de novo lipid synthesis from acetate-derived carbon was reduced in FASN knockdown cells, demonstrating that acetate functions as an acetyl donor to promote lipogenesis and acetate-induced FASN expression plays an important role in lipogenesis (Fig. 5b). Given that elevated lipid synthesis could promote tumour cell survival under hypoxia ${ }^{2,25}$, we carried out CCK8 assay and found that acetate supplement enhanced cell survival under hypoxia, but not normoxia (Fig. 5c). To interrogate whether acetate promoted cancer cell survival through regulating lipogenic genes, we employed specific siRNAs targeting FASN or ACACA, of which the relative low knockdown efficiencies are capable of suppressing acetate-simulated FASN and ACACA mRNA expression to the level similar to unstimulated state (Supplementary Fig. 5a). We found that acetate-induced cell survival under hypoxia was inhibited by knockdown of either FASN or ACACA, indicating that acetate-promoted cell survival was dependent on lipogenesis and the induction of lipogenic gene expression was vital for cell survival (Fig. 5d). Strikingly, we found that inhibition of either FASN or ACACA to the untreated level had no effect on acetate-induced FASN promoter histone acetylation (Supplementary Fig. 5b). We attempted to further dissect whether acetate-induced de novo lipid synthesis is vital for cancer cell cultured under hypoxia. We found that HepG2 and $\mathrm{SkBr} 3$ cells were more sensitive to $\mathrm{C} 75$, a chemical inhibitor of FASN, when cultured under hypoxia (Supplementary Fig. 5c), consistent with previous report that cancer cells were dependent on de novo lipid synthesis under hypoxia 9 . Moreover, C75 treatment abrogated acetate-induced cancer cell survival without affecting FASN and ACACA mRNA levels (Supplementary Fig. 5d,e,f). Consistently, ACSS1/2 knockdown blocked the increased cell survival induced by acetate supplement, indicating that acetate-induced cell survival was ACSS1/2-dependent (Fig. 5e). In addition, we performed Matrigel assay and found that HepG2 cells supplied with acetate exhibited growth advantage over the control group, and this effect was blocked by ACSS1/2 double knockdown (Fig. 5f). Taken together, beyond functioning as a precursor for lipogenesis, acetate also serves as acetyl donor to induce histone acetylation and mRNA expression of lipogenic genes, contributing to the adaptation of cancer cells to hypoxia.

ACSS1/2 positively correlates with FASN expression in HCC. ACSS2 is reported to be upregulated in various human cancers $^{3,5,9}$. ACSS1 is also reported to be increased in hepatocellular carcinoma ${ }^{33}$. Through an analysis of 190 human HCC samples data from the Cancer Genome Atlas (TCGA) ${ }^{34}$, we found a significant correlation between FASN and ACSS2 mRNA levels and no significant correlation between FASN and ACSS1 mRNA levels (Supplementary Fig. 6a). Our aforementioned results demonstrated that acetate could epigenetically regulate FASN expression, which prompts us to examine ACSS1/2, FASN, and $\mathrm{H} 3$ acetylation levels and their correlation in human HCC. We collected 53 pairs of primary human HCC samples with adjacent normal tissues, and determined protein changes (ACSS1, ACSS2 and FASN) and six histone acetylation marks (H3K9ac, H3K14ac, $\mathrm{H} 3 \mathrm{~K} 18 \mathrm{ac}, \mathrm{H} 3 \mathrm{~K} 23 \mathrm{ac}, \mathrm{H} 3 \mathrm{~K} 27 \mathrm{ac}$ and H3K56ac) in all these samples $(n=53)$ (Fig. 6a; Supplementary Fig. 6b). To perform a statistical analysis of the association between ACSS1/2 and histone acetylation, we divided tumour samples into two groups based on ACSS1/2 signature (high versus low). Tumours with 1.5 -fold higher expression of ACSS1 or ACSS2 or both than that of adjacent normal control tissue are grouped into ACSS highexpression tumours (tumour/normal $\geq 1.5, n=26$ ), while ACSS low-expression tumours (tumour/normal $<1.5, n=27$ ) express both ACSS proteins at 1.5 -fold lower than its normal control (Fig. 6b). We found 16 cases with ACSS1 high expression, 8 cases with ACSS2 high expression and 2 cases with both ACSS1 and ACSS2 high expression (Fig. 6b). The higher percentage of ACSS1 high-expression cases than that of ACSS2 fully proves the importance of ACSS1 in acetate utilization by cancer. Our results demonstrate that four out of six histone acetylation marks, including H3K9ac $(P=0.0220)$, H3K14ac $(P=0.0289)$, H3K27ac $(P=0.0034)$ and $\mathrm{H} 3 \mathrm{~K} 56 \mathrm{ac}(P=0.0410)$, are significantly stronger in ACSS high-expression tumours than that from ACSS low-expression tumours with two-tailed unpaired Student's $t$-test (Fig. 6c). In line with this observation, FASN protein level is also significantly upregulated in ACSS high-expression tumours (Fig. 6d). Consistently, we performed immunohistochemistry (IHC) analysis and found that ACSS1/2 expression was positively correlated with $\mathrm{H} 3$ acetylation and FASN expression in human HCC. A representative case was shown in Fig. 6e. By using Spearman correlation analysis, we have evaluated the relationship between histone acetylation and FASN expression. Surprisingly, each and every histone acetylation mark in our analysis exhibits significant positive correlation with FASN expression $(P<0.0001$ for H3K9ac, $P=0.0003$ for H3K14ac, $P=0.0009$ for H3K18ac, $P=0.0385$ for $\mathrm{H} 3 \mathrm{~K} 23 \mathrm{ac}, P<0.0001$ for $\mathrm{H} 3 \mathrm{~K} 27 \mathrm{ac}$ and $P<0.0001$ for H3K56ac; Supplementary Fig. 6c). Collectively, these results strongly support a positive correlation between ACSS1/2, FASN and histone acetylation in human HCC.

\section{Discussion}

Acetyl-CoA is a central metabolite derived mainly from glucose, glutamine and fatty acids in mammals. However, the capability of cancer cells to produce acetyl-CoA from these conventional carbon sources is dramatically decreased under hypoxia ${ }^{3,7}$. Cancer cells, therefore, need alternative carbon sources and 
a
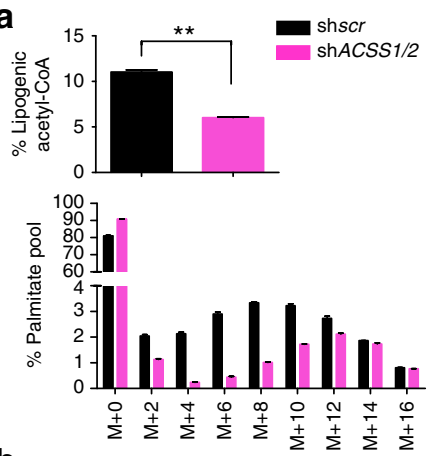

b
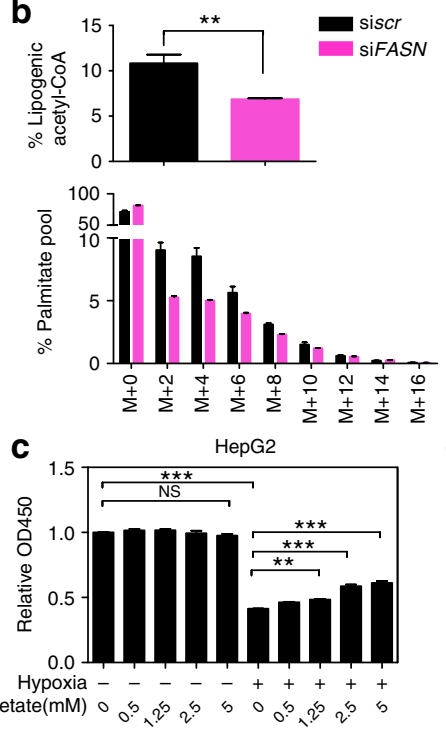

f
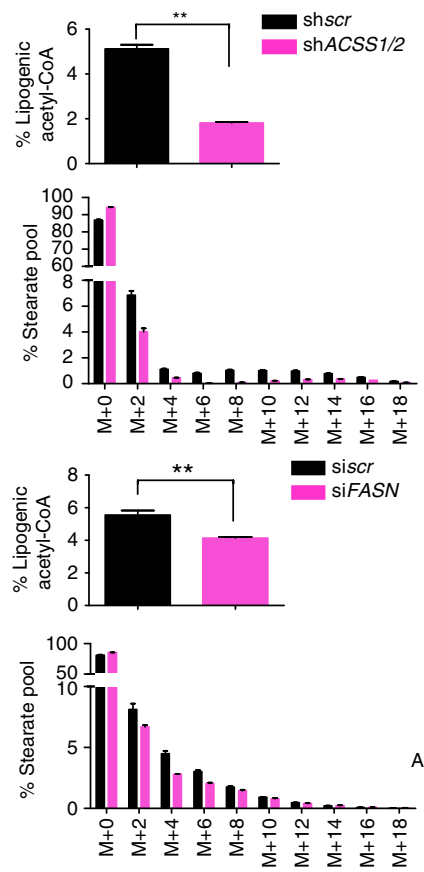

d

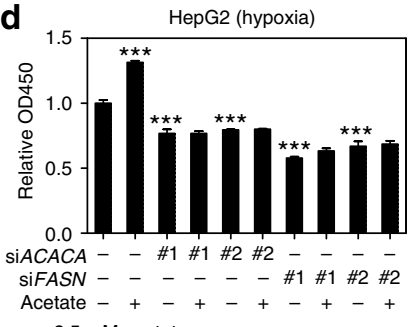

$2.5 \mathrm{mM}$ acetate

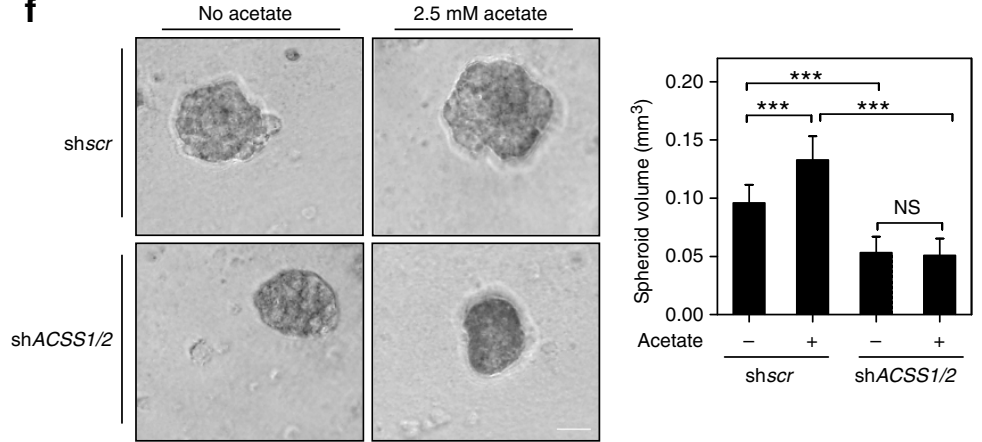

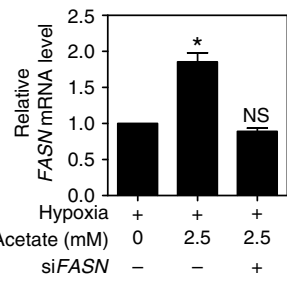

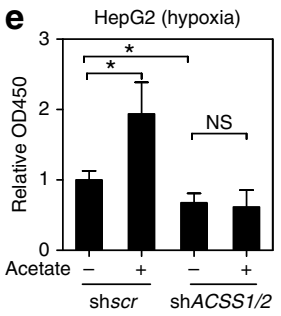

Figure 5 | Epigenetic regulation of de novo lipogenesis by acetate is vital for cell survival under hypoxia. (a) ACSS1/2 mediates acetate-induced palmitate and stearate production. Fractional abundance of palmitate (left) and stearate (right) isotopologues in ACSS1/2 stably double-knockdown HepG2 cells (designated as magenta) compared with that of control cells (designated as black) after $24 \mathrm{~h}$ of culture under hypoxia with $\left[\mathrm{U}-{ }^{13} \mathrm{C}_{2}\right]-$-acetate. Each histogram was presented as mean \pm s.d. of triplicate experiments. The inset shows the percentage of lipogenic acetyl-CoA derived from acetate carbon $\left({ }^{\star \star} P<0.01\right.$; by Student's $t$-test). (b) Fractional abundance of palmitate (left panel) and stearate (middle panel) isotopologues in scramble (siscr) and siFASN HepG2 cells treat as in $\mathbf{a}$. Each histogram was presented as mean \pm s.d. of triplicate experiments. The inset shows the percentage of lipogenic acetyl-CoA derived from acetate carbon. The knockdown efficiency was detected by PCR with reverse transcription (right panel) $\left({ }^{\star \star} P<0.01\right.$; NS, not significant; by Student's t-test). (c)Acetate promotes cell survival under hypoxia. Viability of HepG2 cells treated with indicated concentrations of acetate under normoxia or hypoxia was determined via CCK8 assay. The results were presented as mean \pm s.d. of triplicate experiments $\left({ }^{\star \star} P<0.01 ;{ }^{* \star \star} P<0.001 ; N S\right.$, not significant; by Student's $t$-test). (d) Viability of control or siFSAN or siACACA HepG2 cells treated with or without $2.5 \mathrm{mM}$ acetate under hypoxia was determined via CCK8 assay. The results were presented as mean \pm s.d. of triplicate experiments $\left({ }^{\star \star \star} P<0.001\right.$; by Student's $t$-test). (e) ACSS1/2 mediates the promotion of cell survival by acetate. Viability of shscr or shACSS1/2 HepG2 stable cell lines treated as indicated was determined via CCK 8 assay. The results were presented as mean \pm s.d. of triplicate experiments ( ${ }^{\star} P<0.05$; NS, not significant; by Student's $t$-test). (f) ACSS1/2 mediates cells growth advantage by acetate in Matrigel. Shscr or shACSS1/2 HepG2 cells were plated into the Matrigel bed and cultured with DMEM with $10 \%$ FBS, and $2 \%$ Matrigel for 12 days before the images were taken (left). Scale bar, $0.25 \mathrm{~mm}$. The quantitative data were presented as mean \pm s.d. $(n>3)\left(\right.$ right) ${ }^{\star \star \star} P<0.001 ; N S$, not significant; by Student's $t$-test). 
a
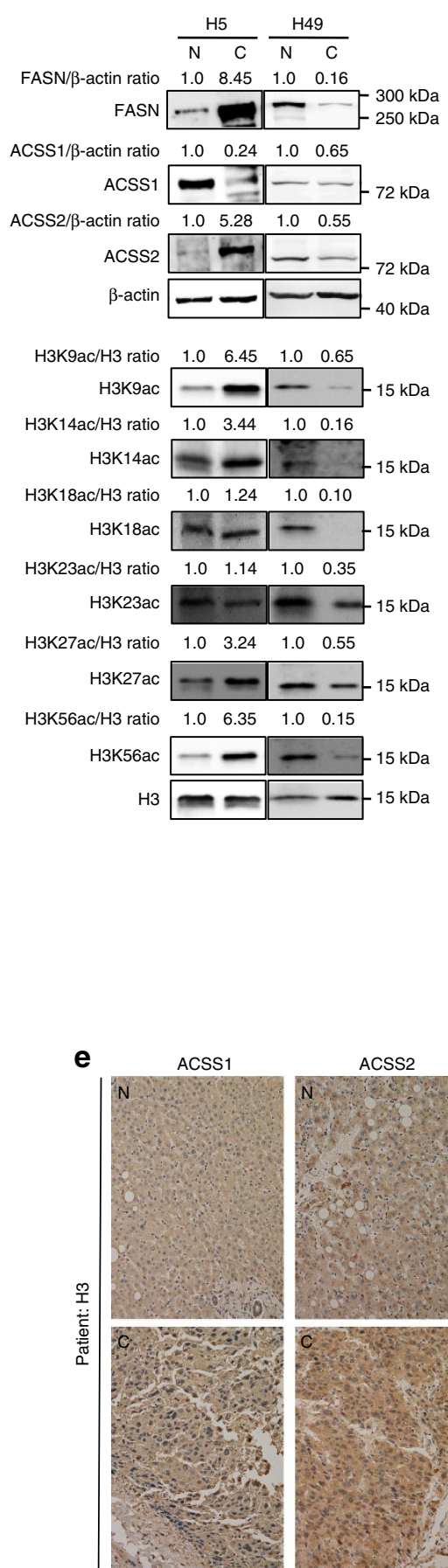

b

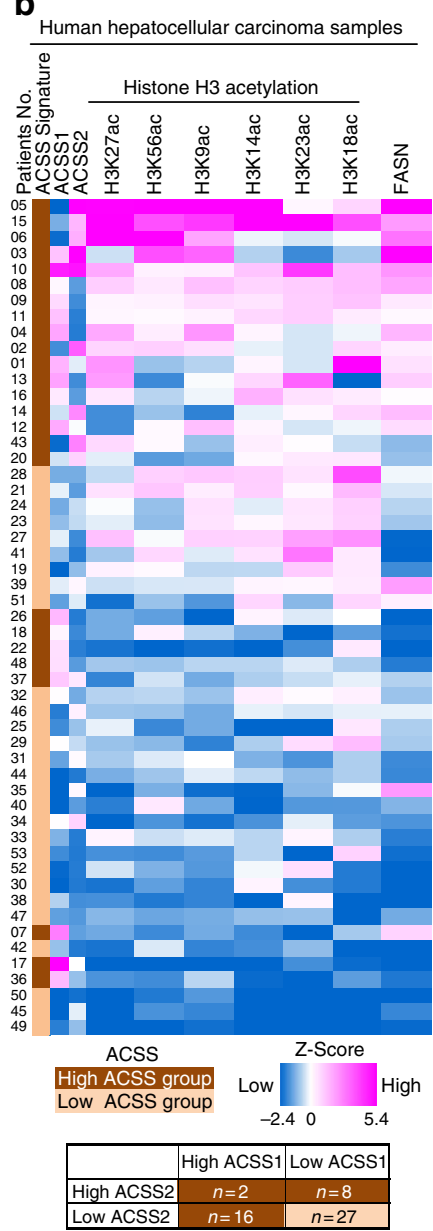

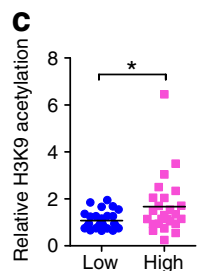

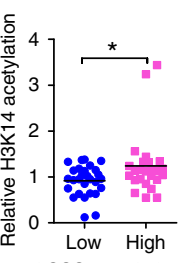

ACSS protein level ACSS protein level
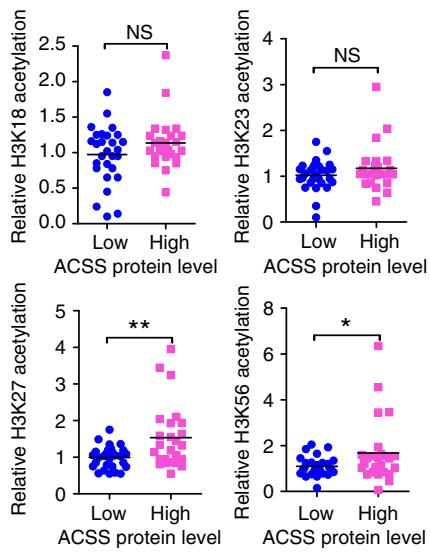

d

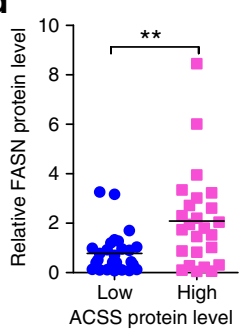

H3K56ac
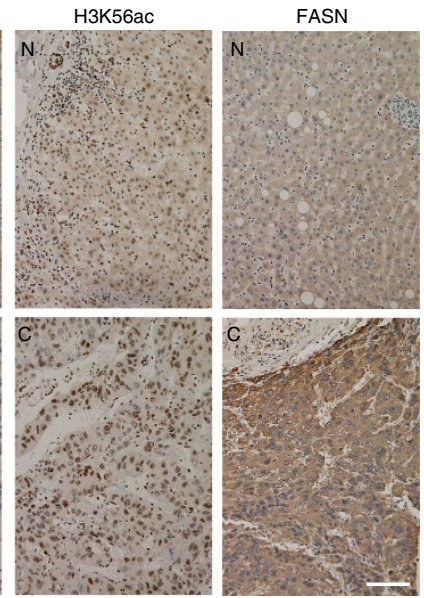

Figure 6 | ACSS1/2 positively correlates with histone acetylation and FASN expression in human hepatocellular carcinoma. (a) FASN, ACSS1 and ACSS2 protein levels (upper panels, normalized against $\beta$-actin) and H3K9, H3K14, H3K18, H3K23, H3K27 and H3K56 acetylation levels (lower panels, normalized against histone $\mathrm{H} 3$ ) in 53 pairs of HCC (each paired with cancerous tissue (designated as $\mathrm{C}$ ) and adjacent normal tissue (designated as $\mathrm{N}$ )) were analysed by western blot. Two pairs of representative samples were shown. For the other 51 pairs of samples, please refer to Supplementary Fig. $6 \mathrm{~b}$. (b) Heatmap of protein expression (ACSS1, ACSS2 and FASN) and histone acetylation levels (H3K9ac, H3K14ac, H3K18ac, H3K23ac, H3K27ac and $\mathrm{H} 3 \mathrm{~K} 56 \mathrm{ac}$ ) in all 53 pairs of HCC. Data were presented as Z-score of relative protein expression or histone acetylation. Tumours with 1.5-fold higher expression of ACSS1 or ACSS2 or both than that of adjacent normal control tissue are grouped into ACSS-high tumours (tumour/normal $\geq 1.5, n=26$ ), while ACSS-low tumours (tumor/normal $<1.5, n=27$ ) express both ACSS proteins at 1.5 -fold lower than its normal control. (c) H3K9ac $(P=0.0220)$, H3K14ac $(P=0.0289)$, H3K27ac $(P=0.0034)$, and H3K56ac $(P=0.0410)$, are significantly stronger in ACSS-high tumours than that in ACSS-low tumours. Statistical analyses were performed with a two-tailed unpaired Student's $t$-test $\left({ }^{\star} P<0.05 ;{ }^{\star \star} P<0.01\right.$; NS, not significant). (d) FASN protein level is significantly upregulated in ACSS-high tumours $(P=0.002)$. Statistical analyses were performed with a two-tailed unpaired Student's $t$-test $\left({ }^{\star *} P<0.01\right)$. (e) Representative immunohistochemical staining results for ACSS1, ACSS2, FASN and H3K9/K27/K56 acetylation in adjacent normal tissue (N) and hepatocellular carcinoma (C). Scale bar, $100 \mu \mathrm{m}$. 
conduct metabolic reprogramming. Clinical studies report the increase in $\left[{ }^{11} \mathrm{C}\right]$-acetate uptake in multiple types of cancers including prostate, liver, lung and brain tumours. Recently, new studies further demonstrate that various tumours consume exogenous acetate to generate acetyl-CoA for lipid synthesis $3,7,9$. These studies support that acetate serves as an important carbon source for cancer cells under unfavourable conditions. The underlying mechanism of how acetate functions as carbon source to fuel tumour growth, however, remains as an open question.

In this study, we elucidate that acetate functions as an epigenetic regulating metabolite to enhance lipid synthesis and to promote tumour survival under hypoxia. First, histone acetylation at $\mathrm{H} 3 \mathrm{~K} 9, \mathrm{H} 3 \mathrm{~K} 27$ and $\mathrm{H} 3 \mathrm{~K} 56$ sites can be stimulated by acetate in both time- and dose-dependent manners under hypoxia in mutiple cancer cell lines. Notably, acetate-derived acetyl-CoA is found to be incorporated not only into fatty acid synthesis but also into acetylated histone peptides. Second, acetate-induced histone acetylation is then associated with $F A S N$ and ACACA promoter regions, which activates FASN and $A C A C A$ expressions under hypoxia. Third, acetate-driven epigenetic regulation is mediated by ACSS1 and ACSS2. Last and most important, acetate-induced lipogenic genes expression is found to promote de novo lipid synthesis and cell survival under hypoxic stress. Collectively, besides its ability to induce fatty acid synthesis as an immediate metabolic precursor, our study reveals a new mechanism of epigenetic regulation by acetate to increase lipid synthesis and promote cell survival under unfavourable conditions (Fig. 7). However, we assess effects of acetate using limited set of cell lines in vitro, it needs further confirmation of the findings using more cell lines. In addition, the experiments in vivo would provide more validation.

Alterations in histone modifications play important roles in transcriptional regulation in cancer cells ${ }^{35}$. H3K9ac, H3K27ac and $\mathrm{H} 3 \mathrm{~K} 56 \mathrm{ac}$, three epigenetic marks of active gene transcription, are found to be altered in various cancers. H3K9 hyperacetylation is associated with specific genes in breast cancer ${ }^{36}$. Similarly, $\mathrm{H} 3 \mathrm{~K} 27$ acetylation is upregulated in colorectal cancer ${ }^{37}$. Intensive investigations also show that H3K56 acetylation is closely related with epigenetic activation and is increased in multiple types of cancer ${ }^{38-43}$. In our work, we found that H3K9, H3K27 and H3K56 acetylation levels were increased in ACSS1/2overexpressed human HCC, showing a significantly positive correlation between ACSS1/2 expression, histone acetylation and FASN expression. ACSS $1 / 2$ upregulation suggests that these cancer cells utilize acetate as both epigenetic metabolite and carbon source to meet their growth needs. These findings validate our model in vivo: ACSS1/2 links histone acetylation with FASN expression and acetate-induced epigenetic regulation may play a pivotal role in human HCC. As the major enzymes in acetate metabolism, ACSS1 and ACSS2 may merit further explorations as a therapeutic target for cancer.

Dysregulation of de novo lipid synthesis is a hallmark of cancer ${ }^{44}$. Increased lipid synthesis fuels cancer engine and contributes to cancer cells survival when facing with stressed conditions, such as hypoxia ${ }^{25,30,44-49}$. Upregulation of FASN is common in many cancers and FASN is targeted for cancer therapy ${ }^{44,49-51}$. FASN inhibition with chemical inhibitors or RNAi can suppress tumour cell survival ${ }^{51}$. FASN upregulation in cancers is in part due to the transcriptional activation by SREBPs ${ }^{44,45,52,53}$. In addition, USP2a was reported to enhance FASN protein stabilization ${ }^{44}$. Our study proves that acetate increases histone acetylation at promoters of lipogenic genes and activates their mRNA expression, without reflecting lipid demand of hypoxic cells. Interestingly, our data show that both ACSS1/2-mediated acetate and ACLY-mediated glucose contribute to histone acetylation at the promoters of hypoxia-induced genes. VEGF or LDHA shows a similar response that occurs in hypoxia alone without acetate addition yet each remains responsive to acetate. This suggests the changes occurring at these promoters that lead to gene activation (presumably recruitment of co-activators) render them more sensitive to acetyl-CoA levels compared with other genes. How recruitment of co-activators modulates acetateinduced lipogenic genes expression is an interesting question.

In conclusion, our study adds a novel mechanism of epigenetic regulation to FASN upregulation. Besides glucose, cancer cells absorb more acetate under hypoxia. Besides functioning as an immediate metabolic precursor, acetate-derived acetyl-CoA increases $\mathrm{H} 3 \mathrm{~K} 9, \mathrm{H} 3 \mathrm{~K} 27$ and $\mathrm{H} 3 \mathrm{~K} 56$ acetylation levels at FASN and ACACA promoter regions, initiating epigenetic regulation of FASN and ACACA. Due to the limitation of our system in distinguishing the role of acetyl-CoA as a substrate rather than an epigenetic regulator, both pathways cannot be separated well and their relative contribution remains unclear. Histone acetylation modulated by acetate status might play a pivotal role in coordinating acetate and glucose availability with the intracellular level of FASN, hence fatty acid synthesis. Taken together, this study demonstrates that acetate, in addition to its ability to induce fatty acid synthesis as an immediate metabolic precursor, also functions as an epigenetic metabolite to promote cancer cell survival under hypoxic stress.

\section{Methods}

Cell culture. PLC-8024 cells were obtained from the Institute of Virology, Chinese Academy of Medical Sciences (Beijing, China). All the other cell lines were obtained from the Cell Bank of Type Culture Collection of Chinese Academy. HepG2, SkBr3, PLC-8024 and DU 145 cells were grown in DMEM medium

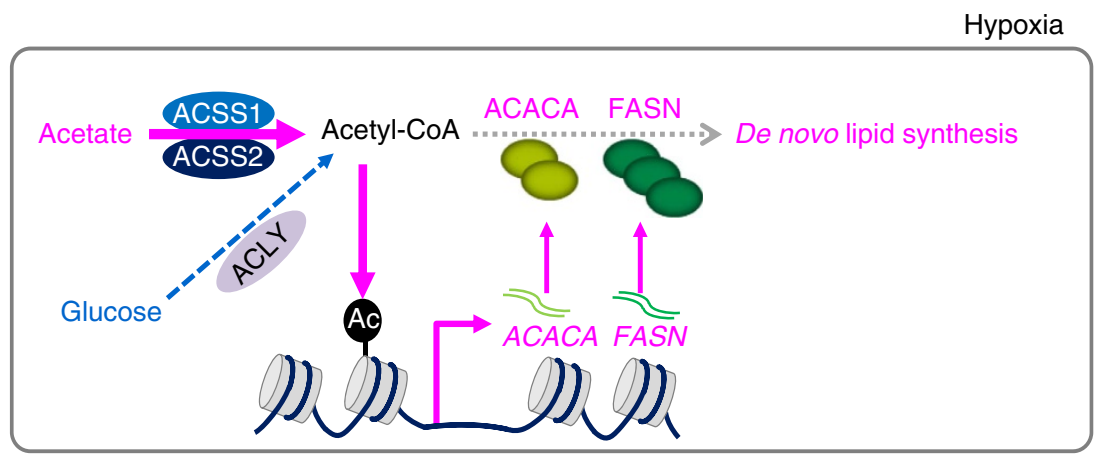

Figure 7 | The model for acetate-induced epigenetic regulation of de novo lipogenesis. In addition to its ability to induce fatty acid synthesis as an immediate metabolic precursor, acetate also functions as an epigenetic metabolite to induce $\mathrm{H} 3$ acetylations in both dose- and time-dependent manners under hypoxia, enhancing $\mathrm{H} 3 \mathrm{~K} 9, \mathrm{H} 3 \mathrm{~K} 27$ and $\mathrm{H} 3 \mathrm{~K} 56$ acetylation levels at FASN and ACACA promoter regions, which upregulates FASN and ACACA expression and increases de novo lipid synthesis to promote tumour cell survival. 
(Gibco, high glucose) with 10\% fetal bovine serum (FBS, biological industries (BI)), and penicillin/streptomycin. A549 cells were grown in F12K medium (Sigma) with $10 \%$ FBS (BI), and penicillin/streptomycin. HT29 cells were cultured in McCoy's 5A medium (Sigma) with 10\% FBS (BI), and penicillin/streptomycin. Mycoplasma contamination check was carried out on cultured cells. Hypoxia condition $\left(1 \% \mathrm{O}_{2}, 94 \% \mathrm{~N}_{2}, 5 \% \mathrm{CO}_{2}\right)$ was achieved by gas chamber (C42, Biospherix).

Antibodies and reagents. Anti-histone H3 (9715S, CST), anti-H3K9 acetyl (13281, Epitomics), anti-H3K27 acetyl (EP865Y, Epitomics), anti-H3K56 acetyl (EPR996Y, Epitomics), anti-ACSS2 (ab133664, Abcam), anti-ACSS1 antibody (17138-1-AP, Proteintech), anti-FASN (C-20140, Santa Cruz), anti-ACLY antibody (1699-1, Epitomics) and anti- $\beta$-actin (a00702, Genescript) antibodies were commercially obtained. ChIP grade antibodies for H3K9 acetyl (ab4441, Abcam), H3K27 acetyl (ab4729, Abcam), H3K56 acetyl (17-10259, Milipore) were commercially obtained.

[U- ${ }^{13} \mathrm{C}_{2}$ ]-Acetate (CLM-440-1, Cambridge Isotope Laboratories), lipoprotein depleted fetal bovine serum (LPDS) (880100, Kalen Biomedical), sodium palmitate (P9767, Sigma), BSA with low fatty acid (0219989925, MP) and TSA (9950, CST) were commercially obtained. Myristic acid (D27, 98\%) as internal standard was obtained from Cambridge Isotope Laboratories (Tewksbury, MA, USA). Certified ACS grade sodium hydroxide was purchased from Standard Chemical and hydrochloric acid was from VWR International LLC (Pennsylvania, USA). Formic acid and BSTFA (with 1\% TMCS) were purchased from Sigma-Aldrich. Methanol and chloroform were obtained from Tedia (Fairfield, USA) and hexane obtained from Duksan Pure Chemicals Co., Ltd (South Korea).

Western blotting. Standard western blot protocols were adopted. The quantification was carried out by subtracting background from the band intensity of western blots by using software ImageQuantTL (GE).

LC-MRM MS quantification of histone acetylation. Histone proteins were extracted and purified as previously described by Gao et $a^{54}$. Briefly, $3 \times 10^{6}$ scramble (shscr) or shACSS1/2 HepG2 cells were cultured in DMEM medium (without glucose or glutamine, Sigma) by adding 10\% dialysed FBS (BI), $2.5 \mathrm{mM}$ $\left[\mathrm{U}-{ }^{13} \mathrm{C}_{2}\right]$-acetate, $5 \mathrm{mM}$ label-free glucose, $2 \mathrm{mM}$ label-free glutamine for $4 \mathrm{~h}$ under hypoxia $\left(1 \% \mathrm{O}_{2}\right)$, followed by lysis with hypotonic lysis buffer $(10 \mathrm{mM}$ Tris- $\mathrm{HCl}$ (pH 8.0), $1 \mathrm{mM} \mathrm{KCl}, 1.5 \mathrm{mM} \mathrm{MgCl}_{2}, 1 \mathrm{mM}$ DTT and protease inhibitors) to isolate nuclei. Crude histone was extracted with $0.4 \mathrm{~N} \mathrm{HCl}$ overnight. After removal of $\mathrm{HCl}$ by ultrafiltration, the crude histone was processed to derivatization in a final condition of $100 \mathrm{mM}$ NHS-propionylate ester (home-made), $25 \mathrm{mM} \mathrm{NH}_{4} \mathrm{HCO}_{3}$, $50 \% \mathrm{ACN}, 50^{\circ} \mathrm{C}$ for $30 \mathrm{~min}$. The proteins were SpeedVac to dryness and digested with trypsin in $25 \mathrm{mM} \mathrm{NH}_{4} \mathrm{HCO}_{3}$ overnight. Finally, the digested peptides were concentrated to dryness again and derivatized for a second round of histone NHS propionylation.

Stable isotope-labelled histone peptides containing various PTMs and chemical derivatization (purity > 95\%) were from New England Peptide LLC (Gardner, MA, USA). Prior to LC-MS analysis, internal standards were mixed with the diluted derivatized histone samples in a ratio of 1:2 in volume. In the final solution, the internal standard was doped as $25 \mathrm{nM}$ stable isotope-labelled histone peptides, while the final concentration of stable isotope-labelled peptides with K27 acetylation was $2.5 \mathrm{nM}$. The peptides were first trapped to a nanoLC trap column at $2 \mathrm{ml} \mathrm{min}{ }^{-1}$ liquid flow that was diverted from the analytical column via a vent valve, while separation was performed by switching the valve to make the trap column in line with a $\mathrm{C} 18$ analytical column with a flow rate of $300 \mathrm{nl} \mathrm{min}^{-1}$. The chromatographic gradient was $5-35 \%$ B from 0 to $45 \mathrm{~min}, 35-80 \%$ B from 45 to 60 min, and maintaining $80 \%$ B for 4 mins (mobile phase A: $0.1 \%$ formic acid in $\mathrm{ddH}_{2} \mathrm{O}$; mobile phase B: $0.1 \%$ formic acid in acetonitrile). Then, the peptides were directly analysed online using QTRAP 6500 mass spectrometer (Applied Biosystems, Singapore) set to multiple reaction monitoring (MRM) in positive electrospray ionization mode. The mass transitions were selected as previously described by Chen $e t a l^{54,55}$, with the addition of transitions corresponding to ${ }^{13} \mathrm{C}_{2}$ labelled acetylated peptides. All MRM data were processed through Skyline software (Washington University in St Louis, St Louis, MO, USA). The quantification was based on the peak area and the measured areas under the curve of indicated product ions were calculated. All data were manually inspected to ensure correct peak detection and accurate integration according to four criteria: (1) the correct $\mathrm{m} / \mathrm{z}$ is selected for both the heavy and light traces of each peptide, and $\left[\mathrm{U}_{-}{ }^{13} \mathrm{C}_{2}\right]$-acetylation for acetylated peptides; (2) the peak shapes are Gaussianlike and do not show excessively jagged appearance; (3) the

retention time of certain peptide is similar between different runs; and (4) the relative contribution of each transition to the total signal is similar between each sample.

RNA extraction and $\mathbf{q P C R}$. Total RNA was extracted with Trizol extraction kit (Invitrogen). qPCR was performed using SYBR Premix Ex Taq (TakaRa) in an ABI 7500 Sequence Detection System (Applied Biosystems). All reactions were performed in triplicate and relative mRNA expression was normalized to $\beta$-actin. All tested primers are listed in Supplementary Table 1.
ChIP-qPCR assays. ChIP-qPCR assays were performed as previously described ${ }^{56}$ Briefly, $1 \times 10^{6}$ HepG2 cells were cross-linked with $1 \%$ paraformaldehyde, lysed and sonicated using the Bioruptor at high-output power setting for eight cycles ( $30 \mathrm{~s} \mathrm{ON}$ and $30 \mathrm{~s}$ OFF). Solubilized chromatin was immunoprecipitated with chip grade antibodies for H3K9 acetyl, H3K27 acetyl, H3K56 acetyl or rabbit IgG (negative control), following that the antibodies were preincubated with protein protein A sepharose overnight at $4{ }^{\circ} \mathrm{C}$. Antibody-chromatin complexes were pulled down by protein A sepharose (Santa Cruz), washed and then eluted. After crosslink reversal in a water bath at $65^{\circ} \mathrm{C}$ overnight and proteinase $\mathrm{K}$ treatment for $2 \mathrm{~h}$ at $55^{\circ} \mathrm{C}$, the immunoprecipitated DNA was extracted with phenol-chloroform, and ethanol precipitated. The DNA fragments were detected by qPCR. Histone acetylation marks were mapped at promoters spanning -3 to $3 \mathrm{~kb}$ of target genes ( $A C A C A, F A S N, A C L Y, V E G F$ and $L D H A$ ). Primers spanning the regions with peaks were adopted for ChIP-qPCR analysis. All tested primers targeting FASN, $A C A C A, A C L Y, L D H A$ and VEGF are listed in Supplementary Table 1.

RNA interference. For RNA interference experiments, si ACSS1 \#1 (EHU040311) and si ACSS2 \#1 (EHU135341) were purchased from Sigma. The others were obtained from commercial synthetic siRNA oligonucleotide (Genepharma, Shanghai).

siACSS1 \#2: 5'-UCACCGUAUUUCAGCAACAGCCGG-3';

siACSS2 \#2: 5'-UAUGCUUGGUGACAGGCUCAUCUCC-3';

siACLY \#1: 5'-GAUCAAACGUCGUGGAAAAUU-3';

siACLY \#2: 5'-GAGGAAGCUGAUGAAUAU-3';

siFASN \#1: 5'-UGGAGCGUAUCUGUGAGAAUU- ${ }^{\prime}$;

siFASN \#2: 5'-AACCCUGAGAUCCCAGCGCUG-3';

siACACA \#1: $5^{\prime}$ - CAAUGGCAUUGCAGCAGUGUU-3';

siACACA \#2: $5^{\prime}$-UAUGAGGUGGAUCGGAGAUUUUU- $3^{\prime}$.

All siRNA transfection experiments were performed with Lipofectamine RNAiMAX Transfection Reagent (Invitrogen) according to the reverse transfection instructions. A volume of $5 \mu \mathrm{l}$ siRNA $(10 \mu \mathrm{M})$ plus with $5 \mu$ l Lipofectamine RNAiMAX per well (six wells) was used. The cell density was $8 \times 10^{5} \mathrm{HepG} 2$ cells per well (six wells). The fresh culture media was exchanged after $24 \mathrm{~h}$. The samples with indicated treatment were collected at $72 \mathrm{~h}$ after transfection. The knockdown efficiency were detected by western blot or qPCR.

Generation of stable knockdown cell lines. The retroviral knockdown constructs of shACSS1 were constructed in pMKO.1-hyg vector as previously described ${ }^{57}$. The lentiviral knockdown constructs of shACSS2 were constructed in pLKO.1-puro vector. The targeting sequences were as follows:

shACSS1\#1: 5'-TCACCGTATTTCAGCAACAGCCGG-3';

shACSS1\#2: $5^{\prime}$-AGGTGGTTATCACCTTCAA-3';

shACSS2\#1: $5^{\prime}$-TACAATGTACTGGATCGAA-3';

shACSS2\#2: 5'-AACGCTTTGAGACAACCTA-3';

The virus was packaged in HEK293T cells by co-transfecting pLKO.1-shACSS2 or pLKO.1-shACSS2 or corresponding vectors (pLKO.1-shscr and pLKO.1-shscr) with package vectors expressing gag and vsvg genes (from vesicular stomatitis virus G). The virus in the culture media was harvested every $24 \mathrm{~h}$ for $48 \mathrm{~h}$ after initial plasmid transfection. For infection, HepG2 cells were infected for $12 \mathrm{~h}$ with pMKO.1-shACSS1 and pMKO-shscr retrovirus with $8 \mu \mathrm{g} \mathrm{ml}^{-1}$ polybrene to increase the infection efficiency and selected in $200 \mu \mathrm{g} \mathrm{ml}^{-1}$ hygromycin B for 1 week first. Then the stable shACSS1 or shscr HepG2 cells were infected with pLKO.1-shACSS2 and pLKO.1-shscr retrovirus and selected in $1 \mu \mathrm{g} \mathrm{ml}^{-1}$ puromycin for 1 week to obtain ACSS1 and 2 double knockdown (shACSS1/2) stable cell lines. The knockdown efficiency was verified by western blot.

NMR spectroscopy. To detect acetate uptake in cancer cells, the cancer cells were grown in DMEM (Gibco, high glucose) supplemented with 10\% FBS (BI) and penicillin/streptomycin. A volume of $200 \mu \mathrm{l}$ of each medium was transferred into a $1.5-\mathrm{ml}$ Eppendorf tube, then $600 \mu \mathrm{l}$ of cold $\mathrm{CD}_{3} \mathrm{OD}$ with $0.09 \mathrm{mg} \mathrm{ml}^{-1}$ trimethylsilyl propionate (TSP) was added to the sample and vortexed for $30 \mathrm{~s}$. The mixture was centrifuged at $20,817 \mathrm{~g}$ for $10 \mathrm{~min}$ at $4^{\circ} \mathrm{C}$. Then $600 \mu \mathrm{l}$ each supernatant was transferred to a standard 5-mm NMR tube for analysis.

To quantitatively analyse acetate in HCC tissues and adjacent normal tissue, $150 \mathrm{mg}$ was weighted for each wet tissue. By adding $700 \mu \mathrm{l}$ methanol and $280 \mu \mathrm{l}$ $\mathrm{H}_{2} \mathrm{O}$ into each sample, homogenization was carried out by using TissueLyser (Qiagen, Germany) for $90 \mathrm{~s}$ at $20 \mathrm{~Hz}$. Subsequently, more $700 \mu \mathrm{l}$ chloroform and $350 \mu \mathrm{H} \mathrm{H}_{2} \mathrm{O}$ were added for further homogenization. The homogenate was centrifuged for $10 \mathrm{~min}$ at $10,621 \mathrm{~g}$ at $4^{\circ} \mathrm{C}$. The supernatant was dried under gentle nitrogen stream. And then the dried residue was added with $600 \mu \mathrm{D}_{2} \mathrm{O}$ containing $0.05 \%$ TSP, vortexed and centrifuged for $8 \mathrm{~min}$ at $20,817 \mathrm{~g}$. The supernatant was removed into a standard 5-mm NMR tube for analysis.

One-dimensional NMR $\left({ }^{1} \mathrm{H}-\mathrm{NMR}\right)$ spectra were obtained on a $600-\mathrm{MHz}$ Bruker Avance III HD spectrometer equipped with a 5-mm PABBO BB-probe. The spectra were acquired with presaturation of the water signal using the Bruker pulse program 'noesygpprld' with a 16-p.p.m., $9,590.793 \mathrm{~Hz}$ width and $4.27 \mathrm{~s}$ acquisition time. All spectra were recorded with 81,920 data points at $25^{\circ} \mathrm{C}$. The time domain data were Fourier-transformed, phase-corrected and baseline-corrected manually. 
The signal intensities were normalized against the intensity of the TSP signal at 0.00 p.p.m.

GC-MS analysis of lipid pool. HepG2 stable cells $\left(5 \times 10^{6}\right)$ were cultured in DMEM medium (without glucose or glutamine, Sigma) by adding $10 \%$ dialysed FBS (BI), $25 \mathrm{mM}$ label-free glucose, $4 \mathrm{mM}$ label-free glutamine and $5 \mathrm{mM}$ $\left[\mathrm{U}_{-}{ }^{13} \mathrm{C}_{2}\right]$-acetate for $24 \mathrm{~h}$ under $1 \% \mathrm{O}_{2}$. Cells were rapidly washed twice with $37^{\circ} \mathrm{C}$ PBS, then $1 \mathrm{ml}$ PBS was added to the dish and quickly detached from the dish using a cell lifter. The liquid-containing cells were transferred into a 2-ml tube and centrifuged at $15,294 \mathrm{~g}$ for $10 \mathrm{~min}$ at $4{ }^{\circ} \mathrm{C}$. The supernatant was discarded and the residue was added to $400 \mu \mathrm{l}$ of cold (80:20 methanol: water) extraction solution for quenching. The sample was vortexed and then stored at $-20^{\circ} \mathrm{C}$ for $1 \mathrm{~h}$. The samples will be vortexed and centrifuged at $15,294 \mathrm{~g}$ for $10 \mathrm{~min}$ at $4{ }^{\circ} \mathrm{C}$. The supernatant will be moved to new tubes for dryness and used for further detection.

The sample preparation of the saponified fatty acids has been described previously ${ }^{7}$. Briefly, $1 \mathrm{ml} \mathrm{MeOH} / \mathrm{H}_{2} \mathrm{O}(1: 1, \mathrm{v} / \mathrm{v})$ solution with $0.1 \mathrm{M} \mathrm{HCl}$ and 1.6 p.p.m. internal standard (myristic acid-D27) in solution was added into cell debris at $0{ }^{\circ} \mathrm{C}$, vortexed for $30 \mathrm{~s}$, followed by addition of $0.5 \mathrm{ml}$ chloroform, and vortexed for $1 \mathrm{~min}$, then centrifuged at $12,000 \mathrm{~g}$ for $5 \mathrm{~min}$ at $4^{\circ} \mathrm{C}$. The resulting chloroform layer was transferred to a new glass vial, and the extract was dried under $\mathrm{N}_{2}$. The desiccation was reconstituted into $1 \mathrm{ml} \mathrm{MeOH} / \mathrm{H}_{2} \mathrm{O}(9: 1, \mathrm{v} / \mathrm{v})$ containing $0.3 \mathrm{M} \mathrm{NaOH}$, and incubated at $80^{\circ} \mathrm{C}$ for $1 \mathrm{~h}$ to saponify the fatty acids. Then $0.1 \mathrm{ml}$ of formic acid was added for acidification, followed by extraction twice with $1 \mathrm{ml}$ hexane. The hexane layer was transferred to an Eppendorf tube for dryness under $\mathrm{N}_{2}$. The dried residue was added to $20 \mu \mathrm{l}$ of pyridine and $80 \mu \mathrm{l}$ of BSTFA (with $1 \%$ TMCS) and vortexed for $30 \mathrm{~s}$, then derivatized at $70^{\circ} \mathrm{C}$ for $1 \mathrm{~h}$. The mixture was vortexed for $30 \mathrm{~s}$ and centrifuged at $12,000 \mathrm{~g}$ for $5 \mathrm{~min}$ at room temperature before GC-MS analysis.

Analysis was performed using an Agilent 7890B gas chromatography system coupled to an Agilent 5977A mass spectrometric detector (MSD, Agilent Technologies). Derivatives were separated using an Agilent 122-5532 UI, DB-5MS capillary column $(30 \mathrm{~m} \times 250 \mu \mathrm{m} \times 0.25 \mu \mathrm{m})$. The injection volume was $1 \mu \mathrm{l}$ in split mode in the ratio 5:1, and the solvent delay time was set to $6 \mathrm{~min}$. The initial oven temperature was set at $100^{\circ} \mathrm{C}$ for $1 \mathrm{~min}$, then ramped to $200^{\circ} \mathrm{C}$ at a rate of $20^{\circ} \mathrm{C} \mathrm{min}-1$, to $260^{\circ} \mathrm{C}$ at a rate of $10^{\circ} \mathrm{C} \mathrm{min}^{-1}$, then to $300^{\circ} \mathrm{C}$ at a rate of $20^{\circ} \mathrm{C} \mathrm{min}^{-1}$ and finally set at $300^{\circ} \mathrm{C}$ for $5 \mathrm{~min}$. Helium was used as a carrier gas at a constant flow rate of $1 \mathrm{ml} \mathrm{min}^{-1}$ through the column. The temperatures of the front inlet, Aux-2 temperature and electron MS source were set at $280^{\circ} \mathrm{C}, 250^{\circ} \mathrm{C}$ and $230^{\circ} \mathrm{C}$, respectively. The electron energy was $70 \mathrm{eV}$, and the mass spectral data were collected in a full-scan mode $(\mathrm{m} / \mathrm{z} 50-600)$. The mass isotopomer distribution of ${ }^{13} \mathrm{C}$-labelled palmitate and stearate was normalized to internal standard and cell number. Natural isotope abundance was corrected using IsoCor ${ }^{58}$

Cell survival assay. shACSS1/2 or shscr HepG2 stable cells were plated in 96-well plates at $1.5 \times 10^{4}$ per well in $200 \mu \mathrm{l}$ of medium treated with indicated conditions. The cell viability was measured after $48 \mathrm{~h}$ treatment by Cell Counting Kit- 8 (Beyotime Biotechnology) according to the manufacturer's instructions. CCK-8 solution was incubated with cells for $1 \mathrm{~h}$ at $37^{\circ} \mathrm{C}$. Absorbances at $450 \mathrm{~nm}$ were collected by ELx800 Universal Microplate Reader (BioTek).

Matrigel assay. Matrigel assay was performed as previously reported with minor modification ${ }^{59}$. Briefly, shscr or shACSS1/2 HepG2 cells were plated in 96-well plates (at 5,000 per well) precoated with 100\% Matrigel bed and cultured in DMEM supplemented with $10 \% \mathrm{FBS}$, and $2 \%$ Matrigel for 12 days. The fresh culture media was exchanged every 2 days. The spheroids were monitored by microscopy. Spheroid size was determined at the times indicated by automated imaging on an inverted microscope (Olympus Fluorescence Microscope IX18).

Hepatocarcinoma cancer samples. Hepatocarcinoma cancer samples were obtained from the 10th People's Hospital, Shanghai (affiliated to Tongii University), with consents from the patients. The procedure related to human subjects was approved by the Ethics Committee of the Institutes of Biomedical Sciences (IBS), Fudan University. Direct immunoblotting was performed as mentioned above.

IHC staining. Human HCC sections were de-paraffinized in xylene and hydrated in graded ethanol, followed by deionized water. Endogenous peroxidase activity was inactivated with $3 \%$ hydrogen peroxidase in methanol for $30 \mathrm{~min}$. Antigen retrieval was performed by boiling the sections in $0.01 \mathrm{M}$ citrate buffer ( $\mathrm{pH}$ 6.0) for $25 \mathrm{~min}$ in microwave oven. The sections were incubated with normal goat serum for 20 min to block non-specific staining and then incubated overnight at $4{ }^{\circ} \mathrm{C}$ with primary antibodies. After incubation for $45 \mathrm{~min}$ with a HRP-conjugated anti-rabbit secondary antibody, the sections were detected using diaminobenzidine (DAB) kit according to the manufacturer's instructions, followed by counterstaining with hematoxylin. The primary antibodies were used as follows: anti-H3K9 acetyl (ab32129, Abcam, 1:150 dilution), anti-H3K27 acetyl (ab45173, Abcam, 1:80 dilution), anti-H3K56 acetyl (ab76307, Abcam, 1:60 dilution), anti-ACSS2 (ab133664, Abcam, 1:50 dilution), anti-ACSS1 antibody (HPA041014, Sigma, 1:50 dilution), and anti-FASN (C-20140, Santa Cruz, 1:40 dilution). The negative controls were performed by omitting the primary antibodies.

Statistical analysis. All data were statistically analysed with two-tailed unpaired Student's $t$-test. The correlation analysis between FASN and ACSS1/2 mRNA levels in 190 human HCC samples from TCGA data base were carried out by using Pearson's coefficient test and the correlation analysis between histone acetylation and FASN expression in human HCC samples by Spearman's correlation test. The values of $P<0.05$ were considered as statistically significant.

Data availability. The analysis result in Supplementary Fig. 6a is based upon data generated by the TCGA Research Network: http://cancergenome.nih.gov/ Data supporting the findings of this study are available within the article and its Supplementary Information files, and from the corresponding author upon request.

\section{References}

1. Wellen, K. E. et al. ATP-citrate lyase links cellular metabolism to histone acetylation. Science 324, 1076-1080 (2009).

2. Metallo, C. M. et al. Reductive glutamine metabolism by IDH1 mediates lipogenesis under hypoxia. Nature 481, 380-384 (2012).

3. Mashimo, T. et al. Acetate is a bioenergetic substrate for human glioblastoma and brain metastases. Cell 159, 1603-1614 (2014).

4. Wellen, K. E. \& Thompson, C. B. A two-way street: reciprocal regulation of metabolism and signalling. Nat. Rev. Mol. Cell Biol. 13, 270-276 (2012).

5. Comerford, S. A. et al. Acetate dependence of tumors. Cell 159, 1591-1602 (2014).

6. Hosios, A. M. \& Vander Heiden, M. G. Acetate metabolism in cancer cells. Cancer Metab. 2, 27-28 (2014).

7. Kamphorst, J. J. et al. Quantitative analysis of acetyl-CoA production in hypoxic cancer cells reveals substantial contribution from acetate. Cancer Metab. 2, 23-30 (2014).

8. Lyssiotis, C. A. \& Cantley, L. C. Acetate fuels the cancer engine. Cell 159, 1492-1494 (2014)

9. Schug, Z. T. et al. Acetyl-CoA synthetase 2 promotes acetate utilization and maintains cancer cell growth under metabolic stress. Cancer Cell 27, 57-71 (2015).

10. Park, J. W. et al. A prospective evaluation of 18F-FDG and 11C-acetate $\mathrm{PET} / \mathrm{CT}$ for detection of primary and metastatic hepatocellular carcinoma. J. Nucl. Med. 49, 1912-1921 (2008).

11. Soloviev, D. et al. PET imaging with 11C-acetate in prostate cancer: a biochemical, radiochemical and clinical perspective. Eur. J. Nucl. Med. Mol. Imaging 35, 942-949 (2008).

12. Tsuchida, T. et al. Grading of brain glioma with 1-11C-acetate PET: comparison with 18F-FDG PET. Nucl. Med. Biol. 35, 171-176 (2008).

13. Oyama, N. et al. 11C-Acetate PET imaging for renal cell carcinoma. Eur. J. Nucl. Med. Mol. Imaging 36, 422-427 (2009).

14. Yoshii, Y. et al. Tumor uptake of radiolabeled acetate reflects the expression of cytosolic acetyl-CoA synthetase: implications for the mechanism of acetate PET. Nucl. Med. Biol. 36, 771-777 (2009).

15. Yun, M. et al. The importance of acetyl coenzyme A synthetase for 11C-acetate uptake and cell survival in hepatocellular carcinoma. J. Nucl. Med. 50, 1222-1228 (2009).

16. Takahashi, H. et al. Nucleocytosolic acetyl-coenzyme a synthetase is required for histone acetylation and global transcription. Mol. Cell 23, 207-217 (2006)

17. Schwer, B. et al. Reversible lysine acetylation controls the activity of the mitochondrial enzyme acetyl-CoA synthetase 2. Proc. Natl Acad. Sci. USA 103, 10224-10229 (2006).

18. North, B. J. \& Sinclair., D. A. Sirtuins: a conserved key unlocking AceCS activity. Trends Biochem. Sci. 32, 1-4 (2007).

19. Yoshii, Y. et al. Cytosolic acetyl-CoA synthetase affected tumor cell survival under hypoxia: the possible function in tumor acetyl-CoA/acetate metabolism. Cancer Sci. 100, 821-827 (2009).

20. Wolin, M. J. \& Miller, T. L. Interactions of microbial populations in cellulose fermentation. Fed. Proc. 42, 109-113 (1983).

21. Pietrocola, F. et al. Acetyl coenzyme A: a central metabolite and second messenger. Cell Metab. 21, 805-821 (2015).

22. Lee, J. V. et al. Akt-dependent metabolic reprogramming regulates tumor cell histone acetylation. Cell Metab. 20, 306-319 (2014).

23. Cai, L. et al. Acetyl-CoA induces cell growth and proliferation by promoting the acetylation of histones at growth genes. Mol. Cell 42, 426-437 (2011).

24. Galdieri, L. \& Vancura, A. Acetyl-CoA carboxylase regulates global histone acetylation. J. Biol. Chem. 287, 23865-23876 (2012).

25. Ackerman, D. \& Simon, M. C. Hypoxia, lipids, and cancer: surviving the harsh tumor microenvironment. Trends Cell Biol. 24, 472-478 (2014).

26. Harris, A. L. Hypoxia--a key regulatory factor in tumour growth. Nat. Rev. Cancer 2, 38-47 (2002). 
27. Mizzen, C. A. \& Allis, C. D. Linking histone acetylation to transcriptional regulation. Cell Mol. Life Sci. 54, 6-20 (1998).

28. Struhl, K. Histone acetylation and transcriptional regulatory mechanisms. Genes Dev. 12, 599-606 (1998).

29. Verdin, E. \& Ott, M. 50 years of protein acetylation: from gene regulation to epigenetics, metabolism and beyond. Nat. Rev. Mol. Cell Biol. 16, 258-264 (2015).

30. Kamphorst, J. J. et al. Hypoxic and Ras-transformed cells support growth by scavenging unsaturated fatty acids from lysophospholipids. Proc. Natl Acad. Sci. USA 110, 8882-8887 (2013).

31. Lin, R. et al. Acetylation stabilizes ATP-citrate lyase to promote lipid biosynthesis and tumor growth. Mol. Cell. 51, 506-518 (2013).

32. Yun, M. et al. The importance of acetyl coenzyme A synthetase for 11C-acetate uptake and cell survival in hepatocellular carcinoma. J. Nucl. Med. 50, 1222-1228 (2009).

33. Bjornson, E. et al. Stratification of hepatocellular carcinoma patients based on acetate utilization. Cell Rep. 13, 2014-2026 (2015).

34. Cerami, E. et al. The cBio cancer genomics portal: an open platform for exploring multidimensional cancer genomics data. Cancer Discov. 2, 401-404 (2012).

35. Suzuki, A. et al. Aberrant transcriptional regulations in cancers: genome, transcriptome and epigenome analysis of lung adenocarcinoma cell lines. Nucleic Acids Res. 110, 13557-13572 (2014).

36. Sadikovic, B. et al. Genome-wide H3K9 histone acetylation profiles are altered in benzopyrene-treated MCF7 breast cancer cells. J. Biol. Chem. 283, 4051-4060 (2008).

37. Karczmarski, J. et al. Histone $\mathrm{H} 3$ lysine 27 acetylation is altered in colon cancer. Clin. Proteomics 11, 24-33 (2014).

38. Williams, S. K. et al. Acetylation in the globular core of histone H3 on lysine-56 promotes chromatin disassembly during transcriptional activation. Proc. Natl Acad. Sci. USA 105, 9000-9005 (2008).

39. $\mathrm{Xu}, \mathrm{F}$. et al. Acetylation in histone $\mathrm{H} 3$ globular domain regulates gene expression in yeast. Cell 121, 375-385 (2005).

40. Vempati, R. K. et al. p300-mediated acetylation of histone H3 lysine 56 functions in DNA damage response in mammals. J. Biol. Chem. 285, 28553-28564 (2010).

41. Lo, K. A. et al. Genome-wide profiling of H3K56 acetylation and transcription factor binding sites in human adipocytes. PLoS ONE 6, e19778-e19789 (2011).

42. Tan, Y. et al. Acetylated histone H3K56 interacts with Oct4 to promote mouse embryonic stem cell pluripotency. Proc. Natl Acad. Sci. USA 110, 11493-11498 (2013).

43. Das, C. et al. CBP/p300-mediated acetylation of histone $\mathrm{H} 3$ on lysine 56. Nature 459, 113-117 (2009).

44. Menendez, J. A. \& Lupu, R. Fatty acid synthase and the lipogenic phenotype in cancer pathogenesis. Nat. Rev. Cancer 7, 763-777 (2007).

45. Furuta, E. et al. Fatty acid synthase gene is up-regulated by hypoxia via activation of Akt and sterol regulatory element binding protein-1. Cancer Res. 68, 1003-1011 (2008)

46. DeBerardinis, R. J., Lum, J. J., Hatzivassiliou, G. \& Thompson, C. B. The biology of cancer: metabolic reprogramming fuels cell growth and proliferation. Cell Metab. 7, 11-20 (2008).

47. Rysman, E. et al. De novo lipogenesis protects cancer cells from free radicals and chemotherapeutics by promoting membrane lipid saturation. Cancer Res. 70, 8117-8126 (2010)

48. Santos, C. R. \& Schulze, A. Lipid metabolism in cancer. FEBS J. 279, 2610-2623 (2012).

49. Jiang, L. et al. Up-regulated FASN expression promotes transcoelomic metastasis of ovarian cancer cell through epithelial-mesenchymal transition. Int. J. Mol. Sci. 15, 11539-11554 (2014).

50. Yang, C. S. et al. Fatty acid synthase inhibition engages a novel caspase-2 regulatory mechanism to induce ovarian cancer cell death. Oncogene 34, 3264-3272 (2015)

51. Kuhajda, F. P. Fatty-acid synthase and human cancer: new perspectives on its role in tumor biology. Nutrition 16, 202-208 (2000).
52. Giandomenico, V., Simonsson, M., Gronroos, E. \& Ericsson, J. Coactivatordependent acetylation stabilizes members of the SREBP family of transcription factors. Mol. Cell Biol. 23, 2587-2599 (2003).

53. Ponugoti, B. et al. SIRT1 deacetylates and inhibits SREBP-1C activity in regulation of hepatic lipid metabolism. J. Biol. Chem. 285, 33959-33970 (2010).

54. Gao, J. et al. Absolute quantification of histone PTM marks by MRM-based LC-MS/MS. Anal. Chem. 86, 9679-9686 (2014).

55. Chen, J. et al. In-gel NHS-propionate derivatization for histone posttranslational modifications analysis in Arabidopsis thaliana. Anal. Chim. Acta. 886, 107-113 (2015).

56. Lan, F. et al. Recognition of unmethylated histone $\mathrm{H} 3$ lysine 4 links BHC80 to LSD1-mediated gene repression. Nature 448, 718-722 (2007).

57. Wang, Y. et al. WT1 recruits TET2 to regulate its target gene expression and suppress leukemia cell proliferation. Mol. Cell. 57, 662-673 (2015).

58. Millard, P. et al. IsoCor: correcting MS data in isotope labeling experiments. Bioinformatics 28, 1294-1296 (2012).

59. Zhang, H. et al. TEAD transcription factors mediate the function of TAZ in cell growth and epithelial-mesenchymal transition. J. Biol. Chem. 284, 13355-13362 (2009).

\section{Acknowledgements}

We thank the members of the Fudan Cancer Metabolism Laboratory for participation throughout this study. We also thank Biomedical Core Facility, Fudan University for technical support. This work was supported by MOST(2015CB910401 and 2013CB911201), NSFC (grant no. 81430057, 81225016, 31271454 and 21377106), Shanghai Key basic research program (12JC1401100), Scholar of 'Dawn' Program of Shanghai Education Commission, Shanghai Outstanding Academic Leader (grant no. 13XD1400600), Cheung Kong Scholar's Program, and the Youth Science and Technology Leading Talent by MOST to Q.Y.L.

\section{Author contributions}

X.G. performed part of the experiments, analysed the data and co-wrote the manuscript. F.R., J.T.L. and C.B.Y. assisted in doing the experiments, data analysis and proofreading the manuscript. S.H.L., J.J.C., P.Y.Y. and S.X.J. contributed to GC-MS analysis of lipid pool and ${ }^{1} \mathrm{H}-\mathrm{NMR}$ spectra of acetate. J.J.C., Y.W., G.Q.Y. and P.Y.Y. contributed to MS analysis. H.B.Y. contributed to analysis of human hepatocarcinoma cancer samples and proofreading the manuscript. D.W. and Y.Y.X. contributed to performing experiments and proofreading the manuscript. Y.L. contributed to analysis of IHC samples. J.C. and W.Q.Y. contributed to proofreading the manuscript. S.H.L., Z.W.C. and P.Y.Y. contributed to design MS experiment and data analysis. Q.Y.L. conceived the idea, designed and supervised the study, analysed the data and co-wrote the manuscript.

\section{Additional information}

Supplementary Information accompanies this paper at http://www.nature.com/ naturecommunications

Competing financial interests: The authors declare no competing financial interests.

Reprints and permission information is available online at http://npg.nature.com/ reprintsandpermissions/

How to cite this article: Gao, X. et al. Acetate functions as an epigenetic metabolite to promote lipid synthesis under hypoxia. Nat. Commun. 7:11960 doi: 10.1038/ncomms11960 (2016).

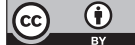

This work is licensed under a Creative Commons Attribution 4.0 International License. The images or other third party material in this article are included in the article's Creative Commons license, unless indicated otherwise in the credit line; if the material is not included under the Creative Commons license, users will need to obtain permission from the license holder to reproduce the material. To view a copy of this license, visit http://creativecommons.org/licenses/by/4.0/ 